\title{
Steady state self-oscillations and chaotic behavior of a controlled electromechanical device by using the first Lyapunov value and the Melnikov theory
}

\author{
Manuel F. Pérez-Polo ${ }^{\mathrm{a}^{*}}$ Manuel Pérez-Molina ${ }^{\mathrm{b}}$,
}

Departamento de Física, Ingeniería de Sistemas y Teoría de la Señal, Escuela Politécnica Superior, Universidad de Alicante.

Campus de San Vicente, Alicante, Spain. Fax 965903682. ' E-mail:

manolo@dfists.ua.es. ${ }^{b}$ E-mail: ma_perez_m@hotmail.com

\begin{abstract}
In this paper regular and chaotic oscillations in a controlled electromechanical transducer are investigated. The nonlinear control laws are defined by an electric tension excitation and an external force applied to the mobile piece of the transducer. The paper shows that an Andronov-Poincaré-Hopf bifurcation appears as long as adequate parameters are chosen for the nonlinear control laws. The stability of the weak focuses associated to such bifurcation is examined according to the sign of the first Lyapunov value, showing that chaotic behavior can arise when the first Lyapunov value is varied harmonically. The appearance of a homoclinic orbit is investigated assuming an approximated model for the device. On the basis of the parametric equations of the homoclinic orbit and the presence of harmonic disturbances on the platform, it is demonstrated that chaotic oscillations can also appear, and they have been examined by means of the Melnikov theory. Chaotic behavior is corroborated by means of the sensitive dependence, Lyapunov exponents and power spectral density, and it is applied to drive the transducer mobile piece to a predefined set point assuming that noise due to the measurement process can appear in the control signals. The steady state error associated to such random noise is eliminated by adding a PI linear controller to the control force. Numerical simulations are used to corroborate the analytical results.
\end{abstract}

\section{Keywords}

Electromechanical transducer, nonlinear control, first Lyapunov value, Melnikov theory, chaos, random noise.

\footnotetext{
$a^{a^{*}}$ Author to whom correspondence should be addressed
} 


\section{TABLE I. NOMENCLATURE}

\section{Variable}

m

B

K

$\mathrm{L}_{1}$

$\mathrm{NL}_{\mathrm{y}}$

SP

c

b

d

$\mathrm{N}$

S

$\mathrm{R}_{\mathrm{g}}$

$\mathrm{R}_{\mathrm{s}}$

$\mu_{0}$

$\mathrm{a}_{\mathrm{m}}$

$\alpha_{1}$

$\alpha_{11}$

$\alpha_{\mathrm{z} 1}$

$\alpha_{\mathrm{z} 2}$

$\alpha_{2}$

$\mathrm{A}_{\mathrm{d}}$

$\omega_{\mathrm{d}}$

$\mathrm{K}_{\mathrm{p}}$

$\mathrm{B}_{\mathrm{f}}$

$\delta$

$\mathrm{K}_{\mathrm{c}}$

$\mathrm{C}_{\mathrm{p}}$

$\tau_{\mathrm{i}}$

$\beta$

$\sigma^{2}$

$\mathrm{r}_{\mathrm{cx}}$

$\mathrm{r}_{\mathrm{cy}}$

$\mathrm{f}_{\text {na }}$

\section{Description}

Mobile mass $(\mathrm{Kg})$

Viscous damping $(\mathrm{N} / \mathrm{m} / \mathrm{s})$

Elastic constant of the spring $(\mathrm{N} / \mathrm{m})$

First Lyapunov value

Lyapunov exponent

Power spectral density

Nonlinear coefficient of the spring $\left(\mathrm{m}^{-2}\right)$

Length of the non-deformed spring $(\mathrm{m})$

Length of the brass component (m)

Number of turns

Cross sectional area $\left(\mathrm{m}^{2}\right)$

Generator resistance $(\Omega)$

Solenoid resistance $(\Omega)$

Vacuum permeability $(\mathrm{H} / \mathrm{m})$

$\mathrm{a}=\left(\mu_{0} \mathrm{~N}^{2} \mathrm{~S}\right) / 2 ; \mathrm{a}_{\mathrm{m}}=\mathrm{a} / \mathrm{m}$

Control law parameter $(\mathrm{A} / \mathrm{m})$

Control law parameter $\left(\mathrm{s}^{-1 / 2}\right)$

Control law parameter $\left(\mathrm{A} / \mathrm{m}^{2} / \mathrm{s}^{2}\right)$

Control parameter $\left(\mathrm{A} / \mathrm{m}^{3} / \mathrm{s}^{3}\right)$

Control law parameter $\left(\mathrm{A}^{-3 / 2} \mathrm{~s}^{-2}\right)$

Disturbance amplitude (rad)

Disturbance frequency ( $\mathrm{rad} / \mathrm{s})$

Proportional constant $(\mathrm{N} / \mathrm{m})$

Viscous damping $(\mathrm{Nm} / \mathrm{s})$

$\delta=\left(\mathrm{B}-\mathrm{B}_{\mathrm{f}}\right) / \mathrm{m}$

$\mathrm{K}_{\mathrm{c}}=\mathrm{cK}\left(\mathrm{m}^{-2} \mathrm{~N} / \mathrm{m}\right)$

Proportional constant of PI controller $\left(\mathrm{s}^{-1}\right)$

Reset time of PI controller $\left(\mathrm{s}^{-2}\right)$

$\beta=\mathrm{K}_{\mathrm{c}} / \mathrm{m}(\mathrm{N} / \mathrm{mkg})$

$\left(\mathrm{K}-\mathrm{K}_{\mathrm{p}}\right) / \mathrm{m}$

Capture zone parameter $(\mathrm{m})$

Capture zone parameter $(\mathrm{m} / \mathrm{s})$

Random noise amplitude
10000

0.05

0.04

Value

0.2

0.02

10

$<0$ ó > 0

100000

$4.10^{-4}$

10

1200

$4 \pi \cdot 10^{-7}$

12.5664

0 -10-20

1-5

0-0.05

0-0.001

10

0.05-0.5

$1-50$

1

0.02

0

$10^{5}$

0.5-1

0.05-0.1

$5 \times 10^{5}$

$\leq 0.02$

$\leq 0.1$

0.005-0.04 


\section{Introduction}

It is known that electromechanical devices are inherently nonlinear, which has allowed them being a rich source of new investigations in vibration systems [1]. These investigations have progressed due to the integrated formulation of mechanical, electric and magnetic circuits [2-7], as well as the combined use of chaos, bifurcation and control theory [8-13]. On the other hand, the appearance of micro electromechanical systems and the developing of new recording systems offer the possibility to examine new interesting dynamical behaviors [14-17].

In this paper we study a device formed by an iron mobile piece actuated by a magnetic field which is generated by an electric circuit, whose voltage is regarded as an external control signal. The other control signal is an external force applied to the mobile piece, which is constrained by a linear damper and a nonlinear spring [1-3]. The control force has a similar structure to a proportional plus derivative (PD) controller. The control voltage is chosen to obtain a proportional (P) controller and to add nonlinear terms that are independent of the system equilibrium points, which are used to stabilize the final position of the device.

Once the control signals have been defined, the equilibrium points and their stability properties depending on the control signals will be analyzed. Such analysis will allow to demonstrate the appearance of a homoclinic orbit with three equilibrium points, being one of them a saddle whereas an Andronov-Poincaré-Hopf bifurcation can arise in the other two equilibrium points [18-20]. The analysis of the bifurcation stability will be carried by calculating the first Lyapunov value [21-24], which will allow to corroborate that a harmonic variation of one parameter involved in the control voltage law may give rise to chaotic dynamic [4-5], [14-15].

Once the bifurcation analysis has been carried out, the Melnikov theory will be applied to obtain sufficient conditions for chaotic behavior [18-19], [24-31]. It will be demonstrated that the chaotic oscillations can be used in connection with the control laws to drive the mobile piece to a predetermined set point with a very small control effort. Finally, we will consider the presence of random noise and the associated steady state error in the final position of the device, which will be removed by adding a linear term formed by a proportional plus integral (PI) controller [28], [32-35]. 


\section{Mathematical model and equilibrium points}

In this section we are going to present the mathematical model of the device as well as the analysis of the equilibrium points and their stability properties.

\subsection{Mathematical model}

The device shown in Fig 1 consists of an electromechanical device used as transducer, which is anchored to a platform that may vibrate harmonically. The transducer working can be described as follows. A voltage generator $\mathrm{V}(\mathrm{t})$ with internal resistance $R_{g}$ is connected to a solenoid with resistance $R_{s}$ and negligible inductance, giving rise to a current $\mathrm{i}(\mathrm{t})$. As a consequence, a magnetic induction field $\mathrm{B}_{\mathrm{e}}$ is generated in a brass piece and another magnetic induction field $B_{\text {eh }}$ appears in the air gap between the solenoid and a mobile piece of mass $\mathrm{m}$, which is connected to the platform by means of a nonlinear spring with a (position-dependent) constant $\mathrm{K}(\mathrm{r})$ and a viscous damping coefficient B [5].

Figure 1

It is assumed that the device is controlled by means of the excitation voltage $V(t)$ and an external force $\mathrm{F}(\mathrm{t})$ applied to the mobile piece. The mathematical model of the transducer is obtained from the Lagrange equations of the system, taking the position $\mathrm{r}_{1}(\mathrm{t})$ of the mobile piece and the electric charge $\mathrm{q}(\mathrm{t})(\mathrm{i}(\mathrm{t})=\mathrm{dq}(\mathrm{t}) / \mathrm{dt})$ as generalized coordinates. The potential energy of the nonlinear spring is defined as:

$$
V_{s}=K\left[\frac{1}{2}\left(r_{1}-b\right)^{2}+\frac{c}{4}\left(r_{1}-b\right)^{4}\right]
$$

where $\mathrm{K}$ and $\mathrm{c}$ are constants, and $\mathrm{b}$ represents the spring length at which no force is exerted on the mobile mass. In particular, if $c=0$ we would have a linear spring.

Assuming that the platform of the device could be oscillating, the total kinetic energy $\mathrm{T}$ of the device is the sum of the kinetic energy of the mobile mass plus the magnetic energy given by: 


$$
T=\frac{1}{2} m\left(\dot{r}_{1}^{2}+r_{1}^{2} \dot{\theta}^{2}\right)+\frac{\mu_{0} S N^{2} \dot{q}^{2}}{2\left(d+r_{1}\right)}
$$

where $\theta=A_{d} \cos \omega_{d} t$ is an external harmonic disturbance due to the platform vibration. The Lagrangian of the system can be written as follows:

$$
L=T-V_{s}=\frac{1}{2} m\left(\dot{r}_{1}^{2}+r_{1}^{2} \dot{\theta}^{2}\right)+\frac{\mu_{0} S N^{2} \dot{q}^{2}}{2\left(d+r_{1}\right)}-K\left[\frac{1}{2}\left(r_{1}-b\right)^{2}+\frac{c}{4}\left(r_{1}-b\right)^{4}\right]
$$

It should be noted that the energy associated to the voltage in the solenoid has not been considered in Eq (3), which implies the assumption that the current $\mathrm{i}(\mathrm{t})$ is almost constant with respect to time. This issue depends on the choice for the control laws and it will be analyzed later.

Assuming that the Rayleigh dissipation function is given by:

$$
F_{R}(\dot{q})=\frac{1}{2} B \dot{r}_{1}^{2}(t)+\frac{1}{2}\left(R_{g}+R_{s}\right) \dot{q}^{2}(t)
$$

and introducing the nomenclature: $\mathrm{R}_{\mathrm{gs}}=\mathrm{R}_{\mathrm{g}}+\mathrm{R}_{\mathrm{s}}, \mathrm{a}=\mu_{0} \mathrm{SN}^{2} / 2$ and $\mathrm{K}_{\mathrm{c}}=\mathrm{cK}$, from the Lagrangian equations and Eqs (3-4) the system equations can be deduced as follows:

$$
\left.\begin{array}{c}
m \ddot{r}_{1}(t)+B \dot{r}_{1}(t)-m r_{1}(t) \dot{\theta}^{2}(t)+K\left[r_{1}(t)-b\right]+K_{c}\left[r_{1}(t)-b\right]^{3}-\frac{a \dot{q}^{2}(t)}{\left[d+r_{1}(t)\right]^{2}}=F(t) \\
\frac{2 a \ddot{q}(t)}{d+r_{1}(t)}-\frac{2 a \dot{q}(t) \dot{r}_{1}(t)}{\left[d+r_{1}(t)\right]^{2}}+R_{g s} \dot{q}(t)=V(t)
\end{array}\right\}
$$

Eqs (5) constitute the mathematical model of the device with harmonic platform disturbances and control signals $F(t)$ and $V(t)$, which will be specified next. To simplify the mathematical treatment, it is convenient to introduce deviation variables defined as follows: 


$$
\left.\begin{array}{c}
z_{1}(t)=r(t)=r_{1}(t)-b ; z_{2}(t)=\dot{r}(t) ; z_{3}(t)=i(t) \\
z_{1}^{\prime}(t)=z_{1}(t)-z_{1 e} ; z_{2}^{\prime}(t)=z_{2}(t) ; z_{3}^{\prime}(t)=z_{3}(t)-i_{0 e} \\
z_{1 e}=r_{1 e}-b \Rightarrow z_{1}^{\prime}(t)=z_{1}(t)-r_{1 e}+b \Rightarrow z_{1}^{\prime}(t)=r_{1}(t)-b-r_{1 e}+b=r_{1}(t)-r_{1 e}
\end{array}\right\}
$$

where $\mathrm{z}_{1 \mathrm{e}}$ and $\mathrm{i}_{0 \mathrm{e}}$ are the equilibrium position of the mobile mass and current equilibrium respectively, which can be obtained from equilibrium equations once the control signals $F(t)$ and $V(t)$ have been specified. Substituting Eqs (6) into Eqs (5), taking $\mathrm{a}_{\mathrm{m}}=\mathrm{a} / \mathrm{m}$ and assuming that the harmonic disturbance is $\theta(t)=A_{d} \cos \omega_{d} t$, it is possible to deduce the mathematical model in terms of deviation variables as [5], [28]:

$$
\begin{aligned}
& \dot{z}_{1}^{\prime}(t)=z_{2}^{\prime}(t) \\
& \begin{array}{c}
\dot{z}_{2}^{\prime}(t)=-\frac{K}{m}\left[z_{1}^{\prime}(t)+z_{1 e}\right]-\frac{B}{m} z_{2}^{\prime}(t)-\frac{K_{c}}{m}\left[z_{1}^{\prime}(t)+z_{1 e}\right]^{3}-\frac{a_{m}\left[z_{3}^{\prime}(t)+i_{0 e}\right]^{3}}{\left[d_{b}+z_{1}^{\prime}(t)+z_{1 e}\right]^{2}} \\
+\left[z_{1}^{\prime}(t)+z_{1 e}+b\right] \omega_{d}^{2} A_{d}^{2} \sin ^{2} \omega_{d} t+\frac{1}{m} F(t) \\
\dot{z}_{3}^{\prime}(t)=\frac{z_{2}^{\prime}(t)\left[z_{3}^{\prime}(t)+i_{0 e}\right]}{d_{b}+z_{1}^{\prime}(t)+z_{1 e}}-\left[\frac{d_{b}+z_{1}^{\prime}(t)+z_{1 e}}{2 a}\right] R_{g s}\left[z_{3}^{\prime}(t)+i_{e}\right]+\left[\frac{d_{b}+z_{1}^{\prime}(t)+z_{1 e}}{2 a}\right] V(t)
\end{array}
\end{aligned}
$$

The control signals $\mathrm{F}(\mathrm{t})$ and $\mathrm{V}(\mathrm{t})$ are chosen as:

$$
\begin{gathered}
F(t)=F_{0}+K_{p}\left[z_{1}^{\prime}(t)+z_{1 e}\right]+B_{f} z_{2}^{\prime}(t)-f_{d} m\left[z_{1}^{\prime}(t)+z_{1 e}+b\right] A_{d}^{2} \omega_{d}^{2} \sin ^{2} \omega_{d} t \\
V(t)=R_{g s}\left[z_{3}^{\prime}(t)+i_{0 e}\right]-\frac{2 a z_{2}^{\prime}(t)\left[z_{3}^{\prime}(t)+i_{0 e}\right]}{\left[d_{b}+z_{1 e}+z_{1}^{\prime}(t)\right]}+\left[\frac{2 a}{d_{b}+z_{1 e}+z_{1}^{\prime}(t)}\right] f\left[z_{2}^{\prime}(t), z_{3}^{\prime}(t)\right]
\end{gathered}
$$

where the nonlinear function $f\left[z_{2}^{\prime}(t), z_{3}^{\prime}(t)\right]$ is defined by:

$$
f\left[z_{2}^{\prime}(t), z_{3}^{\prime}(t)\right]=\alpha_{1} z_{2}^{\prime}(t)-\alpha_{11}^{2} z_{3}^{\prime}(t)-\alpha_{z 1} z_{2}^{\prime 2}(t)-\alpha_{z 2} z_{2}^{\prime 3}(t)-\alpha_{2}^{2} z_{3}^{\prime 3}(t)
$$

The first three terms of the control force $F(t)$ can be considered as a classic PD (proportional + derivative) control with a bias given by $F_{0}$ whose effect will be analyzed later, and the other term could be considered as feedforward control where $f_{d}$ 
is a disturbance factor that accounts for the harmonic base excitation of the system [45]. It should be noticed that the disturbance is canceled by the control law when $f_{d}=1$.

The control tension $V(t)$ represents the voltage in the resistance $R_{g s}=R_{g}+R_{s}$, where $R_{s}$ compensates the voltage originated by the magnetic-mechanical coupling (i.e. the voltage due to the mobile mass movement). The following terms can be identified in Eq (10): a derivative controller with constant $\alpha_{1}$, a proportional controller with constant $-\alpha_{11}^{2}$, a nonlinear frictional term formed by the constants $-\alpha_{\mathrm{z} 1}$ and $-\alpha_{\mathrm{z} 2}$ and a nonlinear term multiplied by $\alpha_{2}^{2}$ to stabilize the current $\mathrm{z}_{3}{ }_{3}(\mathrm{t})$. The previous arrangement of Eq (10) can be justified as follows. Substituting Eqs (8), (9) and (10) into Eqs (7) and introduction the notation:

$$
\sigma^{2}=\frac{K-K_{p}}{m} ; \beta=\frac{K_{c}}{m} ; \delta=\frac{B-B_{f}}{m}
$$

Eqs (7) can be rewritten as follows:

$$
\begin{aligned}
& \dot{z}_{1}^{\prime}(t)=z_{2}^{\prime}(t) \\
& \begin{aligned}
& \dot{z}_{2}^{\prime}(t)=- \sigma^{2}\left[z_{1}^{\prime}(t)+z_{1 e}\right]-\delta z_{2}^{\prime}(t)-\beta\left[z_{1}^{\prime}(t)+z_{1 e}\right]^{3}-\frac{a_{m}\left[z_{3}^{\prime}(t)+i_{0 e}\right]^{2}}{\left[d_{b}+z_{1}^{\prime}(t)+z_{1 e}\right]^{2}} \\
& \quad+\frac{F_{0}}{m}+\left(1-f_{d}\right)\left[z_{1}^{\prime}(t)+z_{1 e}+b\right] A_{d}^{2} \omega_{d}^{2} \sin ^{2} \omega_{d} t \\
& \dot{z}_{3}^{\prime}(t)=\alpha_{1} z_{2}^{\prime}(t)-\alpha_{11}^{2} z_{3}^{\prime}(t)-\alpha_{z 1} z_{2}^{\prime 2}(t)-\alpha_{z 2} z_{2}^{\prime 3}(t)-\alpha_{2}^{2} z_{3}^{\prime 3}(t)
\end{aligned}
\end{aligned}
$$

Assuming that $\alpha_{1}=0, \alpha_{z 1}=0$ and $\alpha_{z 2}=0$, a differential equation with $z_{3}^{\prime}(t)$ as unknown is obtained from the third equation of Eqs (12), and the solution for such differential equation is given by:

$$
z_{3}^{\prime}(t)=\frac{\alpha_{11} C_{1} \exp \left(-\alpha_{11}^{2} t\right)}{\sqrt{1-\alpha_{2}^{2} C_{1}^{2} \exp \left(-2 \alpha_{11}^{2} t\right)}} ; C_{1}=\frac{z_{30}^{\prime}}{\sqrt{\alpha_{11}^{2}+\alpha_{2}^{2} z_{30}^{\prime 2}}}
$$

where $z^{\prime}{ }_{30}$ is an arbitrary initial condition. On the other hand, the mean value of $z^{\prime}{ }_{3}(t)$ can be calculated from $\mathrm{Eq}(13)$ as follows: 


$$
\vec{z}_{3}^{\prime}(t)=\frac{1}{t_{m}} \int_{0}^{t_{m}} z_{3}^{\prime}(t) \mathrm{d} t=\frac{z_{30}^{\prime}}{\alpha_{11}^{2} t_{m}}-\frac{1}{\alpha_{11} t_{m}}\left(\frac{\alpha_{11} C_{1} \exp \left(-\alpha_{11}^{2} t_{m}\right)}{\sqrt{1-\alpha_{2}^{2} C_{1}^{2} \exp \left(-2 \alpha_{11}^{2} t_{m}\right)}}\right)
$$

It should be noticed that $\mathrm{z}_{3}{ }_{3}(\mathrm{t}) \rightarrow 0$ for $\mathrm{t} \rightarrow \infty$ when $\alpha_{11}>1$ and $\alpha_{2}>1$. So according to Eqs (13) and (14), the current in the resistance $\mathrm{R}_{\mathrm{gs}}$ can be regarded as approximately constant. This implies that the voltage in the inductor can be considered as negligible in accordance with the kinetic energy defined by Eq (2).

\subsection{Equilibrium points}

The equilibrium points are deduced from Eqs (12) in the absence of external harmonic disturbance. Since we are using deviation variables, in the equilibrium points we must have that $\dot{z}_{1}^{\prime}=0, \dot{z}_{2}^{\prime}=0, \dot{z}_{3}^{\prime}=0 ; z_{1}^{\prime}=0, z_{2}^{\prime}=0, z_{3}^{\prime}=0$, so from Eqs (6) and (12) we obtain the equilibrium condition given by:

$$
f\left(r_{1 e}\right)=\left(d+r_{1 e}\right)^{2}\left\{\left[\sigma^{2}\left(r_{1 e}-b\right)+\beta\left(r_{1 e}-b\right)^{3}\right]-f_{0}\right\}+a_{m} i_{0 e}^{2}=0
$$

where $f_{0}=F_{0} / m$ and the equilibrium current is calculated from Eq (15) as $i_{0 e}=V_{0} / R_{g s}$, being $\mathrm{V}_{0}$ a fixed value for the applied tension. Fig 2 shows the value of $\mathrm{f}\left(\mathrm{r}_{1 \mathrm{e}}\right)$, which has been plotted as a function of $r_{1 e}$ taking $F_{0}=7.1343 \mathrm{~N}$-this particular value has been chosen to obtain several equilibrium points- and $\mathrm{V}_{0}$ as a parameter.

Figure 2

The roots of Eq (15) are the crossing points of the horizontal line $f\left(r_{1 e}\right)=0$ with the curves shown in Fig 2, but the equilibrium points $r_{1 e}$ of Eqs (12) are only the positive roots of $\mathrm{Eq}$ (15) (see Fig 1). In particular, the roots of $\mathrm{Eq}$ (15) for $\mathrm{V}_{0}=[200$, 210, 220, 230, 240] are indicated in the rows of the following matrix M: 


$$
M=\left[\begin{array}{ccccc}
1.1401 \mathrm{e}-001 & -5.6216 \mathrm{e}-002 & 5.6858 \mathrm{e}-002+ & 5.6858 \mathrm{e}-002- & \\
& & 1.6375 \mathrm{e}-002 \mathrm{i} & 1.6375 \mathrm{e}-002 \mathrm{i} & -8.8718 \mathrm{e}-003 \\
1.1303 \mathrm{e}-001 & -5.6903 \mathrm{e}-002 & 5.6371 \mathrm{e}-002+ & 5.6371 \mathrm{e}-002- & \\
& & 1.0689 \mathrm{e}-002 \mathrm{i} & 1.0689 \mathrm{e}-002 \mathrm{i} & -8.8718 \mathrm{e}-003 \\
1.1190 \mathrm{e}-001 & -5.7581 \mathrm{e}-002 & 6.3897 \mathrm{e}-002 & 4.7602 \mathrm{e}-002 & -5.8186 \mathrm{e}-003 \\
-5.8251 \mathrm{e}-002 & 1.1056 \mathrm{e}-001 & 7.1819 \mathrm{e}-002 & 3.7970 \mathrm{e}-002 & -2.0947 \mathrm{e}-003 \\
-5.8912 \mathrm{e}-002 & 1.0890 \mathrm{e}-001 & 7.7530 \mathrm{e}-002 & 2.9486 \mathrm{e}-002 & 2.9927 \mathrm{e}-003
\end{array}\right]
$$

Depending on the values of $V_{0}$, the following cases can appear: i) For $V_{0}=240$ $\mathrm{V}$ (last row of matrix $\mathrm{M}$ ) there are five real roots being four of them positive and the other one negative (which cannot be an equilibrium point), so in this case we have four equilibrium points $\mathrm{P}_{1}, \mathrm{P}_{2}, \mathrm{P}_{3}{ }^{\prime}$ and $\mathrm{P}_{4}{ }^{\prime}$. ii) For $\mathrm{V}_{0}=230 \mathrm{~V}$ and $220 \mathrm{~V}$ the system has only three equilibrium points, which in the case of $\mathrm{V}_{0}=230 \mathrm{~V}$ are $\mathrm{P}_{1}, \mathrm{P}_{2}$ and $\mathrm{P}_{3}$. iii) The case of two equilibrium points may appear when the horizontal line $f\left(r_{1 e}\right)=0$ is tangent to a curve giving two positive values for $r_{1 e}$ and the rest of them being negative or conjugate complex. iv) For $\mathrm{V}_{0}=210 \mathrm{~V}$ and $200 \mathrm{~V}$, the polynomial $\mathrm{f}\left(\mathrm{r}_{1 \mathrm{e}}\right)$ has three real roots and two conjugate complex ones. Since two of the real roots are negative, in this case we have only one equilibrium point.

It should be noted that the case of four equilibrium points appears for high values of $F_{0}$ and $V_{0}$, the case of two equilibrium points is difficult to obtain in practice since small disturbances would destroy the tangency, and with only one equilibrium point the possible applications of the device are reduced. Consequently, in this paper, we will only consider values for $\mathrm{F}_{0}$ and $\mathrm{V}_{0}$ which give rise to three equilibrium points.

Once the equilibrium points of Eqs (12) have been obtained from the roots of Eq (15), it should be remarked that the function $f\left[z_{2}^{\prime}(t), z_{3}^{\prime}(t)\right]$ is independent of the position $r_{1 e}$, since the deviation variables $z_{2}{ }_{2}(t)$ and $z_{3}{ }_{3}(t)$ are independent of $r_{1 e}$ (see Eqs (6)). For example, equilibrium points $\mathrm{P}_{1}, \mathrm{P}_{2}$ and $\mathrm{P}_{3}$ of Fig 2 -each of them with different values of $r_{1}$ - have been obtained for $\mathrm{V}_{0}=230 \mathrm{~V}$ and $\mathrm{F}_{0}=7.1343 \mathrm{~N}$, and consequently all of them have the same equilibrium current $i_{0 e}=V_{0} / R_{g s}$. 
In view of Fig 2 it is deduced that one, two, three of four equilibrium point can appear depending on the voltage $\mathrm{V}_{0}$. The stability of the equilibrium points is obtained from the eigenvalues of the Jacobian matrix of the linearized system around the local coordinates of each equilibrium point. Since in Eq (12) we are using deviation variables, the equilibrium points are translated to the origin, i.e. in the equilibrium we have that $z_{1}^{\prime}=0, z_{2}^{\prime}=0, z_{3}^{\prime}=0$ and thus the Jacobian matrix can be written as follows:

$$
\mathbf{J}=\left[\begin{array}{ccc}
0 & 1 & 0 \\
-\sigma^{2}-3 \beta z_{1 e}^{2}+\frac{2 a_{m} i_{0 e}^{2}}{\left(d_{b}+z_{1 e}\right)^{3}} & \delta & -\frac{2 a_{m} i_{0 e}}{\left(d_{b}+z_{1 e}\right)^{2}} \\
0 & \alpha_{1} & -\alpha_{11}
\end{array}\right]
$$

where $d_{b}=d+b$ and $d_{b}+z_{1 e}=d+b+r_{1 e}-b=d+r_{1 e}$. Introducing the notation:

$$
a_{21}=-\sigma^{2}-3 \beta\left(r_{1 e}-b\right)^{2}+\frac{2 a_{m} i_{0 e}}{\left(d+r_{1 e}\right)^{2}} ; a_{23}=-\frac{2 a_{m} i_{0 e}^{2}}{\left(d+r_{1 e}\right)^{3}}
$$

and assuming that $\delta=0$ (i.e. $B=B_{\mathrm{f}}$ ), the eigenvalues of the Jacobian matrix $\mathrm{J}$ can be obtained according to $\mathrm{Eq}(17)$ as:

$$
|\lambda \mathbf{I}-\mathbf{J}|=\lambda^{3}+\alpha_{11}^{2} \lambda^{2}+\omega^{2} \lambda+b_{21} \alpha_{11}^{2}=0
$$

where

$$
\begin{gathered}
\omega^{2}=-\left(a_{21}+\alpha_{1} a_{23}\right)=\sigma^{2}+3 \beta\left(r_{1 e}-b\right)^{2}-\frac{2 a_{m} i_{0 e}^{2}}{\left(d+r_{1 e}\right)^{3}}+\alpha_{1} \frac{2 a_{m} i_{0 e}}{\left(d+r_{1 e}\right)^{2}} \\
b_{21}=-a_{21}=\sigma^{2}+3 \beta\left(r_{1 e}-b\right)^{2}-\frac{2 a_{m} i_{0 e}^{2}}{\left(d+r_{1 e}\right)^{3}}
\end{gathered}
$$

By applying the Routh-Hurwitz criterion to Eq (19) and taking into account Eqs (20), it is deduced that $b_{21}=\omega^{2}$ for $\alpha_{1}=0$, and assuming that $\sigma^{2}+3 \beta z_{1 e}^{2}>2 a_{m} i_{0 e}^{2} /\left(d_{b}+z_{1 e}\right)^{3}$ the eigenvalues of the equilibrium point (which depend on $\mathrm{r}_{1 \mathrm{e}}$ ) will be $\lambda_{1}=-\alpha_{11}^{2} ; \lambda_{2,3}= \pm j \omega$ and consequently an Andronov-Poincaré-Hopf bifurcation will appear [22-24]. This condition can only be fulfilled at the equilibrium 
points where the derivative of $f\left(r_{1 e}\right)$ is positive (points $P_{1}$ and $P_{3}$ of Fig 2), whereas at the equilibrium point $\mathrm{P}_{2}$ the derivative is negative and therefore $\sigma^{2}+3 \beta z_{1 e}^{2}<2 a_{m} i_{0 e}^{2} /\left(d_{b}+z_{1 e}\right)^{3}$, so it is an unstable saddle.

\section{Chaotic behaviors from the Andronov-Poincaré-Hopf bifurcation}

It is well known that an Andronov-Poincaré-Hopf bifurcation is associated to the so called weak focus, whose stability depends on the sign of the first Lyapunov value. Nevertheless, when the stability type changes with the appearance or disappearance of small periodic orbits encircling the equilibrium point, a weak focus appears. In this case, it is not possible to know if the equilibrium point will be stable or unstable [1819], [21-24].

For second order systems it is well known that it is possible to find a polynomic change of state variables which removes the even terms in the Taylor series of the nonlinear terms. In this case, the system can be written in polar coordinates as [18-19], [2123], [30]:

$$
\begin{aligned}
& \dot{r}=L_{1} r^{3}+\ldots+L_{k} r^{2 k+1}+\mathrm{O}\left(r^{2 k+1}\right) \\
& \dot{\theta}=\omega+\Omega_{1} r^{2}+\ldots+\Omega_{k} r^{2 k}+\mathrm{O}\left(r^{2 k}\right)
\end{aligned}
$$

Equation (21) is called normal form of the system, and the coefficients $L_{k}$ are called Lyapunov values, whose calculation requires knowing the Taylor series expansion of the non-linear terms up to order $(2 k+1)$. When the Lyapunov values up to order $k-1$ are null (i.e. $L_{1}=L_{2}=\ldots . L_{k-1}=0$ ), a new change of coordinates allows one to obtain the orbital normal form, from which it is possible to demonstrate that the (spirallike) trajectory converges to an stable equilibrium point as $t \rightarrow \infty$ if $L_{k}<0$. On the other hand, when $L_{k}>0$ the equilibrium point is called unstable weak focus because trajectories starting close to equilibrium point spiral away as $t \rightarrow \infty$. If $L_{k}=0$, the calculation of the $L_{k+1}$ value will be necessary in order to know the stability of the equilibrium point. To calculate $L_{k}$ in a high dimensional case, a simplified system on the centre manifold must be calculated considering up to third order terms. 
From the previous discussion, the determination of the first Lyapunov value implies obtaining the Taylor series expansion and expansion of non-linear terms of Eqs (12) up to order three. For this purpose, it will be necessary to carry out a set of previous steps in order to rewrite equations (12) in an appropriate form to determine the first Lyapunov value. These steps are summarized as follows.

\section{Step 1.}

Taking $\delta=0, \alpha_{1}=0$ and assuming that $\mathrm{A}_{\mathrm{d}}=0$, the Taylor series expands of the nonlinear terms in the second Eq (12) allow to rewrite the system equations as:

$$
\begin{aligned}
& \dot{z}_{1}^{\prime}(t)=z_{2}^{\prime}(t) \\
& \dot{z}_{2}^{\prime}(t)=-\sigma^{2}\left[z_{1}^{\prime}(t)+z_{1 e}\right]-\beta\left[z_{1}^{\prime}(t)+z_{1 e}\right]^{3}- \\
& -a_{m}\left[z_{3}^{\prime}(t)+i_{0 e}\right]^{2} \sum_{n=0}^{\infty}(-1)^{n} \frac{(n+1) !}{\left(d_{b}+z_{1 e}\right)^{n+2}} z_{1}^{\prime n}(t)+\frac{F_{0}}{m} \\
& \dot{z}_{3}^{\prime}(t)=-\alpha_{11}^{2} z_{3}^{\prime}(t)-\alpha_{z 1} z_{2}^{\prime 2}(t)-\alpha_{z 2} z_{2}^{\prime 3}(t)-\alpha_{2}^{2} z_{3}^{\prime 3}(t)
\end{aligned}
$$

Taking into account the equilibrium condition given in Eq (15) and considering terms up to order 3 , the system equations (22) can be written in a matrix form as follows:

$$
\left[\begin{array}{l}
\dot{z}_{1}^{\prime}(t) \\
\dot{z}_{2}^{\prime}(t) \\
\dot{z}_{3}^{\prime}(t)
\end{array}\right]=\left[\begin{array}{ccc}
0 & 1 & 0 \\
a_{21} & 0 & a_{23} \\
0 & 0 & -\alpha_{11}^{2}
\end{array}\right]\left[\begin{array}{l}
z_{1}^{\prime}(t) \\
z_{2}^{\prime}(t) \\
z_{3}^{\prime}(t)
\end{array}\right]+\left[\begin{array}{c}
0 \\
f_{2}^{\prime}\left[z_{1}^{\prime}(t), z_{2}^{\prime}(t)\right] \\
f_{3}^{\prime}\left[z_{1}^{\prime}(t), z_{2}^{\prime}(t)\right]
\end{array}\right]
$$

where

$$
\begin{aligned}
& f_{2}^{\prime}\left[z_{1}^{\prime}(t), z_{2}^{\prime}(t)\right]=C_{11} z_{1}^{\prime 2}(t)+C_{33} z_{3}^{\prime 2}(t)+C_{13} z_{1}^{\prime}(t) z_{3}^{\prime}(t)+C_{111} z_{1}^{\prime 3}(t)+ \\
& +C_{133} z_{1}^{\prime}(t) z_{3}^{\prime 2}(t)+C_{113} z_{1}^{\prime 2}(t) z_{3}^{\prime}(t)+\ldots . . \\
& f_{3}^{\prime}\left[z_{1}^{\prime}(t), z_{2}^{\prime}(t)\right]=-\alpha_{z 1} z_{2}^{\prime 2}(t)-\alpha_{z 2} z_{2}^{\prime 3}(t)-\alpha_{2}^{2} z_{3}^{\prime 3}(t)
\end{aligned}
$$

and

$$
\begin{gathered}
C_{11}=-3 \beta z_{1 e}-\frac{2 a_{m} i_{0 e}^{2}}{\left(d_{b}+z_{1 e}\right)^{4}} ; C_{13}=\frac{4 a_{m} i_{0 e}^{2}}{\left(d_{b}+z_{1 e}\right)^{3}} \\
C_{33}=-\frac{a_{m}}{\left(d_{b}+z_{1 e}\right)^{2}} ; C_{111}=-\beta+\frac{4 a_{m} i_{0 e}^{2}}{\left(d_{b}+z_{1 e}\right)^{5}}
\end{gathered}
$$


As we will see later, the rest of the coefficients $C_{i j k}$ do not influence the calculation of the first Lyapunov value.

Remark. It has been proved by Wang [21] that if the functions $f_{2}^{\prime}$ and $f_{3}^{\prime}$ given in Eq (24) are both homogeneous polynomials of a degree $m$ such that $2 \mathrm{k} /(\mathrm{m}-1)$ is not an integer, then $\mathrm{L}_{\mathrm{k}}$ must be zero. In our case, we have that $\mathrm{k}=1$ and $\mathrm{m}=3$, so $2 \mathrm{k} /(\mathrm{m}-1)=1$ and consequently the first Lyapunov value $\mathrm{L}_{1} \neq 0$ can be used to analyze the stability of the weak focus.

Step 2.

Let $P$ be the matrix that transforms the linear part of Eqs (23) into its Jordan canonical form. For details see Refs [18-20], [23-24]. Under the linear transformation

$$
z^{\prime}=\mathbf{P} \bar{x} \Rightarrow\left[\begin{array}{c}
z_{1}^{\prime} \\
z_{2}^{\prime} \\
z_{3}^{\prime}
\end{array}\right]=\left[\begin{array}{ccc}
0 & 1 & 1 \\
\omega & 0 & -\alpha_{11}^{2} \\
0 & 0 & \left(\alpha_{11}^{4}-a_{21}\right) / a_{23}
\end{array}\right]\left[\begin{array}{l}
x \\
y \\
z
\end{array}\right]
$$

equation (28) becomes

$$
\left[\begin{array}{c}
\dot{x}(t) \\
\dot{y}(t) \\
\dot{z}(t)
\end{array}\right]=\left[\begin{array}{ccc}
0 & -\omega & 0 \\
\omega & 0 & 0 \\
0 & 0 & -\alpha_{11}^{2}
\end{array}\right]\left[\begin{array}{l}
x(t) \\
y(t) \\
z(t)
\end{array}\right]+\mathbf{P}^{-1}\left[\begin{array}{c}
0 \\
f_{2}^{\prime}[x(t), y(t), z(t)] \\
f_{3}^{\prime}[x(t), y(t), z(t)]
\end{array}\right]
$$

where

$$
\begin{gathered}
\mathbf{P}^{-1}=\frac{-a_{23}}{\omega\left(\alpha_{11}^{4}-a_{21}\right)}\left[\begin{array}{ccc}
0 & -\left(\alpha_{11}^{4}-a_{21}\right) / a_{23} & -\alpha_{11}^{2} \\
-\omega\left(\alpha_{11}^{4}-a_{21}\right) / a_{23} & 0 & \omega \\
0 & 0 & -\omega
\end{array}\right] \\
f_{2}^{\prime}[x(t), y(t), z(t)]=f_{2}[\bar{x}(t)]=C_{11}(y+z)^{2}+C_{33}\left(\frac{\alpha_{11}^{4}-a_{21}}{a_{23}}\right)^{2} z^{2}+C_{13}\left(\frac{\alpha_{11}^{4}-a_{21}}{a_{23}}\right)(y+z) z \\
+C_{111}(y+z)^{3}+C_{133}\left(\frac{\alpha_{11}^{4}-a_{21}}{a_{23}}\right)^{2}(y+z) z^{2}+C_{113}\left(\frac{\alpha_{11}^{4}-a_{21}}{a_{23}}\right)(y+z)^{2} z+\ldots \ldots \\
f_{3}^{\prime}[x(t), y(t), z(t)]=f_{3}[\bar{x}(t)]=-\alpha_{z 1}\left(\omega x-\alpha_{11}^{2} z\right)^{2}-\alpha_{z 2}\left(\omega x-\alpha_{11}^{2} z\right)^{3}-\alpha_{2}^{2}\left(\frac{\alpha_{11}^{4}-a_{21}}{a_{23}}\right)^{3} z^{3}
\end{gathered}
$$




$$
\mathbf{P}^{-1}\left[\begin{array}{c}
0 \\
f_{2}(\bar{x}) \\
f_{3}(\bar{x})
\end{array}\right]=\left[\begin{array}{c}
\bar{f}_{1}(\bar{x}) \\
\overline{f_{2}}(\bar{x}) \\
\bar{f}_{3}(\bar{x})
\end{array}\right]=\left[\begin{array}{c}
\frac{1}{\omega} f_{2}(\bar{x})+\frac{\alpha_{11}^{2} a_{23}}{\omega\left(\alpha_{11}^{4}-a_{21}\right)} f_{3}(\bar{x}) \\
-\frac{a_{23}}{\alpha_{11}^{4}-a_{21}} f_{3}(\bar{x}) \\
\frac{a_{23}}{\alpha_{11}^{4}-a_{21}} f_{3}(\bar{x})
\end{array}\right]
$$

Step 3.

We now simplify equations (27)-(29) as much as possible using the center manifold theorem in order to obtain a reduced two-dimensional system in a neighbourhood around the equilibrium point [4-5]. In the center manifold the variables $z$ is approximated by:

$$
z=h(x, y)=\bar{b}_{11} x^{2}+\bar{b}_{22} y^{2}+\bar{b}_{12} x y
$$

Coefficients $b_{i j}(\mathrm{i}, \mathrm{j}=1,2)$ are determined by substituting equation (34) into the equation which defines the center manifold, which is given by:

$$
\mathbf{D}_{\overline{\mathbf{x}}} h(x, y)\left[\overline{\mathbf{A}} \overline{\mathbf{x}}_{\mathbf{m}}+\overline{\mathbf{f}}(x, y, h(x, y))\right]=\mathbf{B} h(x, y)+\overline{\mathbf{g}}(x, y, h(x, y))
$$

where

$$
\begin{gathered}
\overline{\mathbf{x}}_{\mathbf{m}}=\left[\begin{array}{l}
x \\
y
\end{array}\right] ; \overline{\mathbf{A}}=\left[\begin{array}{cc}
0 & -\omega \\
\omega & 0
\end{array}\right] ; \overline{\mathbf{f}}(x, y, h(x, y))=\left[\begin{array}{l}
\bar{f}_{1}(x, y, h(x, y)) \\
\bar{f}_{2}(x, y, h(x, y))
\end{array}\right] \\
B=-\alpha_{11}^{2} ; \bar{g}(x, y, h(x, y))=\bar{f}_{3} ; \quad \mathbf{D}_{\overline{\mathbf{x}}} h(x, y)=\left[\frac{\partial h(x, y)}{\partial x}, \frac{\partial h(x, y)}{\partial y}\right]
\end{gathered}
$$

It should be noticed that the coefficients up to order three in Eqs (31) do not influence the calculation of coefficients $b_{i j}$ of Eq (30), so coefficients $C_{133}, C_{113}$ and $\alpha_{2}^{2}$ can be omitted in Eq (30). Taking into account Eqs (31) and (32) and equating the terms in $x^{2}$, $x y, y^{2}$ in the left and right sides of equation (31), the coefficients $b_{i j}$ can be computed by using the following equation:

$$
\left[\begin{array}{ccc}
\alpha_{11}^{2} & 0 & \omega \\
0 & \alpha_{11}^{2} & -\omega \\
-2 \omega & -2 \omega & \alpha_{11}^{2}
\end{array}\right]\left[\begin{array}{l}
\bar{b}_{11} \\
\bar{b}_{22} \\
\bar{b}_{12}
\end{array}\right]=\left[\begin{array}{c}
-\left(a_{23} \omega^{2} /\left(\alpha_{11}^{4}-a_{21}\right)\right) \alpha_{z 1} \\
0 \\
0
\end{array}\right]
$$


The equations of the system reduced to the center manifold can be written as follows:

$$
\left[\begin{array}{c}
\dot{x} \\
\dot{y}
\end{array}\right]=\left[\begin{array}{cc}
0 & -\omega \\
\omega & 0
\end{array}\right]\left[\begin{array}{l}
x \\
y
\end{array}\right]+\left[\begin{array}{l}
\bar{f}_{1}(x, y, h(x, y)) \\
\bar{f}_{2}(x, y, h(x, y))
\end{array}\right]
$$

In order to simplify the next calculations, the following nomenclature has been introduced:

$$
\begin{gathered}
f_{2}\left(\bar{x}_{m}\right)=p_{02} y^{2}+p_{21} x^{2} y+p_{12} x y^{2}+p_{03} y^{3} \\
C_{x y}=2 C_{11}+C_{13}\left(\frac{\alpha_{11}^{4}-a_{21}}{a_{23}}\right) \\
p_{02}=C_{11} ; p_{21}=C_{x y} \bar{b}_{11} ; p_{12}=C_{x y} \bar{b}_{12} ; p_{03}=C_{x y} \bar{b}_{22}+C_{111} \\
f_{3}\left(\bar{x}_{m}\right)=q_{20} x^{2}+q_{21} x^{2} y+q_{12} x y^{2}+q_{30} x^{3} \\
q_{20}=-\alpha_{z 1} \omega^{2} ; q_{21}=2 \omega \alpha_{11}^{2} \alpha_{z 1} \bar{b}_{12} ; q_{12}=2 \omega \alpha_{11}^{2} \alpha_{z 1} \bar{b}_{22} ; q_{30}=2 \omega \alpha_{11}^{2} \alpha_{z 1} \bar{b}_{11}
\end{gathered}
$$

Taking into account Eqs (29), (35) and (36), the functions $\bar{f}_{i}(x, y, h(x, y))(\mathrm{i}=1,2)$ of Eq (34) can be written as follows:

$$
\begin{gathered}
\bar{f}_{1}(x, y)=a_{20} x^{2}+a_{02} y^{2}+a_{21} x^{2} y+a_{12} x y^{2}+a_{30} x^{3}+a_{03} y^{3} \\
\bar{f}_{2}(x, y)=b_{20} x^{2}+b_{21} x^{2} y+b_{12} x y^{2}+b_{30} x^{3} \\
C_{\alpha 1}=\frac{\alpha_{11}^{2} a_{23}}{\omega\left(\alpha_{11}^{4}-a_{21}\right)} ; C_{\alpha 2}=\frac{a_{23}}{\alpha_{11}^{4}-a_{21}} ; a_{20}=C_{\alpha 1} q_{20}=-\alpha_{z 1} \omega^{2} C_{\alpha 1} \\
a_{02}=\frac{p_{02}}{\omega}=\frac{C_{11}}{\omega} ; a_{21}=\frac{p_{21}}{\omega}+C_{\alpha 1} q_{21}=\frac{C_{x y} b_{11}}{\omega}+C_{\alpha 1} 2 \omega \alpha_{11}^{2} \alpha_{z 1} \bar{b}_{12} \\
a_{12}=\frac{p_{12}}{\omega}+C_{\alpha 1} q_{12}=\frac{C_{x y} \bar{b}_{12}}{\omega}+C_{\alpha 1} 2 \omega \alpha_{11}^{2} \alpha_{z 1} \bar{b}_{22} \\
a_{30}=C_{\alpha 1} q_{30}=C_{\alpha 1}\left(2 \omega \alpha_{11}^{2} \alpha_{z 1} \bar{b}_{11}-\alpha_{z 2} \omega^{3}\right) \\
a_{03}=\frac{p_{03}}{\omega}=\frac{1}{\omega}\left(C_{x y} \bar{b}_{22}+C_{111}\right) ; b_{20}=C_{\alpha 2} q_{20}=C_{\alpha 2} \alpha_{z 1} \omega^{2} \\
b_{21}=-C_{\alpha 2} q_{21}=-C_{\alpha 2} 2 \omega \alpha_{11}^{2} \alpha_{z 1} \bar{b}_{12} ; b_{12}=-C_{\alpha 2} q_{12}=-C_{\alpha 2} 2 \omega \alpha_{11}^{2} \alpha_{z 1} \bar{b}_{22} \\
b_{30}=-C_{\alpha 2} q_{30}=-C_{\alpha 2}\left(2 \omega \alpha_{11}^{2} \alpha_{z 1} \bar{b}_{11}-\alpha_{z 2} \omega^{3}\right)
\end{gathered}
$$

Step 4.

Considering a system of the form given by Eqs (34)-(37), the first Lyapunov value $L_{1}$ can be expressed as [18-19], [23-24]: 


$$
\begin{gathered}
L_{1}=\frac{1}{16}\left[f_{x x x}^{1}+f_{x y y}^{1}+f_{x x y}^{2}+f_{y y y}^{2}\right]+ \\
+\frac{1}{16 \omega}\left[f_{x y}^{1}\left(f_{x x}^{1}+f_{y y}^{1}\right)-f_{x y}^{2}\left(f_{x x}^{2}+f_{y y}^{2}\right)-f_{x x}^{1} f_{x x}^{2}+f_{y y}^{1} f_{y y}^{2}\right]
\end{gathered}
$$

where $f^{1} \equiv \bar{f}_{1} ; f^{2} \equiv \bar{f}_{2}$ and the sub-indexes $x y \ldots$ mean partial derivatives evaluated at the equilibrium point, which in our case is the origin since we are using deviation variables. Applying equation (37) to equation (38), the value of $L_{l}$ is:

$$
L_{1}=\frac{1}{16}\left(6 a_{30}+2 a_{12}+2 b_{12}\right)-\frac{1}{16 \omega} 2 a_{20} 2 b_{20}
$$

To verify the previous calculations, the parameter $\omega$ has been plotted as a function of the equilibrium point $r_{1 e}$ in Fig 3. It should be noticed that there are values of $r_{1 e}$ for which there are not pure imaginary eigenvalues, since $\sigma^{2}+3 \beta z_{1 e}^{2}<2 a_{m} i_{0 e}^{2} /\left(d_{b}+z_{1 e}\right)^{3}$ and consequently the Andronov-Poincaré-Hopf bifurcation does not appear for such values. In Fig 3 b an equilibrium curve similar to the one shown in Fig 2 has been plotted for $\mathrm{V}_{0}=227.2167 \mathrm{~V}$ and $\mathrm{i}_{0 \mathrm{e}}=0.1878 \mathrm{~A}$. In this case, the transducer has three equilibrium points $\mathrm{P}_{1}, \mathrm{P}_{2}$ and $\mathrm{P}_{3}$, whose values $P_{e}$ and their corresponding eigenvalues $E_{e}$ (taking $\left.\alpha_{11}=1\right)$ are the following ones:

$$
\begin{aligned}
& P_{e}\left[P_{1}(0.1110) \quad P_{2}(0.0700) \quad P_{3}(0.0403)\right] \\
& E_{e}[ \pm 34.9976 j,-1 \pm 21.6980,-1 \quad \pm 26.373 j,-1]
\end{aligned}
$$

From Eq (40) It is deduced that $\mathrm{P}_{2}(0.0700)$ with eigenvalues $( \pm 21.6980,-1)$ is a saddle and therefore is unreachable, whereas $\mathrm{P}_{1}$ and $\mathrm{P}_{3}$ are weak focuses and their stability depends on the sign of the first Lyapunov value given by $\mathrm{Eq}$ (39). If $\mathrm{L}_{1}<0$, the equilibrium point $\left(\mathrm{P}_{1}\right.$ or $\left.\mathrm{P}_{3}\right)$ will be stable, and when $\mathrm{L}_{1}>0$ the same equilibrium point will be unstable. It is interesting to remark that the case which we are analyzing $\left(\mathrm{V}_{0}=\right.$ 227.2167 $\mathrm{V}$ and $\mathrm{F}_{0}=7.1343 \mathrm{~N}$ ) is similar to the one plotted in Fig 2 for $\mathrm{V}_{0}=230 \mathrm{~V}$ and $\mathrm{F}_{0}=7.1343 \mathrm{~N}$. So, among the five roots given by Eq (15), only three of them (the positive ones) are equilibrium points. 
Figure 3

Fig 4 shows the variation of the first Lyapunov value (Eq (39)) as a function of the equilibrium points plotted in Fig 3 a, which allows to appreciate the stability change for the equilibrium points with pure imaginary eigenvalues as a function of $\alpha_{z 1}$. For $\alpha_{z 1}$ $=0.05, \alpha_{11}=1$ and $\alpha_{z 2}=0.001$, the equilibrium point $\mathrm{P}_{1}$ is a stable weak focus and the equilibrium point $\mathrm{P}_{3}$ is an unstable one. The apparent convergence of the curves at $\mathrm{r}=$ 0.03 is a visual effect due to the scale that has been used to represent high values of $\mathrm{L}_{1}$. Fig 5 shows two cases of weak stable and unstable focus plotted in the phase plane. In Fig 5 a, the equilibrium points $\mathrm{P}_{1}$ and $\mathrm{P}_{3}$ are unstable and stable respectively, since their corresponding first Lyapunov values are $\mathrm{L}_{1}\left(\mathrm{P}_{1}\right)>0$ and $\mathrm{L}_{1}\left(\mathrm{P}_{3}\right)<0$. Fig 5 b shows a case in which the equilibrium points $\mathrm{P}_{1}$ and $\mathrm{P}_{3}$ are both unstable. According to Fig 1 it can be appreciated that the mobile piece reaches the end position in contact with the non-ferromagnetic piece, which gives rise to a rebound in which the velocity vector changes its direction abruptly in a short time lapse. For this purpose, a restitution coefficient between 0.1 and 0.3 has been assumed in the rebound. It should be noted that in this case the system reaches a limit cycle after an initial transient.

Figure 4

Figure 5

To analyze the dynamical behavior of the transducer, Eqs (12) have been simulated taking $\mathrm{f}_{\mathrm{d}}=1$, i.e. assuming that there are not external harmonic disturbances on the platform. It is assumed that the control law parameter $\alpha_{z 1}$ is varied as $\alpha_{z 1}=\alpha_{L z 1} \cos \omega_{z} t$, where the equilibrium points $\mathrm{P}_{1}, \mathrm{P}_{2}$ and $\mathrm{P}_{3}$ are independent of $\alpha_{z 1}$ (see Eqs (12)) and thus they remain constant. In this case, if there is a sign change in the first Lyapunov values $L_{1}\left(P_{1}\right)$ and $L_{1}\left(P_{3}\right)$ of the equilibrium points $P_{1}$ and $P_{3}$ respectively, the system will jump from one equilibrium point to another. Fig 6 a shows the sign variations for $\mathrm{L}_{1}\left(\mathrm{P}_{1}\right)$ and $\mathrm{L}_{1}\left(\mathrm{P}_{3}\right)$, whereas in Fig $6 \mathrm{~b}$ the simulation results for the transducer position clearly corroborate that the system cannot remain oscillating around an unstable weak focus, and thus it must jump to the other equilibrium point (with a negative first Lyapunov value). At the initial time instant the transducer is at point $\mathrm{P}_{2}$ (which is a saddle), and it holds that $\mathrm{L}_{1}\left(\mathrm{P}_{1}\right)<0$ and $\mathrm{L}_{1}\left(\mathrm{P}_{3}\right)>0$. Consequently, the system jumps to 
point $P_{1}$ and remains oscillating around this point while $t<t_{1}$. When $t$ exceeds $t_{1}$, the stability of $\mathrm{P}_{1}$ and $\mathrm{P}_{3}$ is then interchanged, i.e. $\mathrm{L}_{1}\left(\mathrm{P}_{1}\right)>0$ and $\mathrm{L}_{1}\left(\mathrm{P}_{3}\right)<0$. The RungeKutta integration method has been used taking a simulation time $t_{m}=20 \mathrm{~s}$, a simulation step $\mathrm{T}=5.10^{-4} \mathrm{~s}$, initial conditions: $\left[0,0,10^{-4}, 0\right]$ and $\mathrm{r}_{1 \mathrm{e}}=0.07 \mathrm{~m}$.

Figure 6

The results obtained from the analysis of the first Lyapunov value allow us to conclude that if $L_{1}$ vary according to a harmonic law the system could reach chaotic behavior without external harmonic disturbances. To research this issue, figure 7 shows the results obtained by simulating the system equations (12) during large times. Figs $7 \mathrm{a}$ and $7 \mathrm{~b}$ respectively depict the variations of the magnetic field in the air gap and the velocity of the transducer mobile mass. In Figs $7 \mathrm{c}$ and $7 \mathrm{~d}$ the values for the current and its derivative corroborate that the voltage in the solenoid can be considered as negligible. It should be remarked that the simulation values are acceptable from a physical viewpoint.

Figure 7

Figs. $8 \mathrm{a}$ and $\mathrm{b}$ show the appearance of sensitive dependence when the system is simulated with two very close initial conditions (at approximately $t=90 \mathrm{~s}$ the initial error has increased in 7 orders of magnitude). On the other hand, Figs $8 \mathrm{c}$ and $8 \mathrm{~d}$ show that the variation of the control signals given by Eqs (14)-(16) leads to physically feasible values. The fourth order Runge-Kutta integration method has been used taking initial conditions $\left[0,0,10^{-4}, 0\right]$, a simulation time $t_{m}=120 \mathrm{~s}$ and a simulation step $\mathrm{T}=5.10^{-4}$.

Figure 8

Fig 9 a shows the strange attractor in the phase plane $r_{1}(t)-d r_{1}(t) / d t$ as well as the equilibrium points $\mathrm{P}_{1}$ and $\mathrm{P}_{3}$. To verify the hypothesis of chaotic behavior, the Lyapunov exponents have been plotted as a function of the time in Fig $9 \mathrm{~b}$. The values obtained at $\mathrm{t}_{\mathrm{m}}$ $=120 \mathrm{~s}$ are $\mathrm{L}_{\mathrm{y}}(120)=[0.4181,-0.4288,-0.99370]$, for which the presence of a positive exponent is an indicator of chaos. The Runge-Kutta-Fehlberg integration method has been used with simulation steps between $10^{-4}$ and $5.10^{-4} \mathrm{~s}$. The zero value in $\mathrm{L}_{\mathrm{y}}$ is due to the auxiliary angular variable $z_{4}{ }_{4}(t)$ defined by [5], [28]: 


$$
\frac{\mathrm{d} z_{4}^{\prime}(t)}{\mathrm{d} t}=\omega_{z} ; z_{4}^{\prime}(t) \bmod 2 \pi
$$

which has been introduced to transform Eqs (12) into an autonomous system when $\alpha_{z 1}$ is varied harmonically. Fig $9 \mathrm{c}$ shows the sum of Lyapunov exponents and the divergence of the vector field given by Eqs (12), both of them as a function of the time. The mean value of the vector field divergence and the sum of the Lyapunov exponents are related by [5], [23,24], [36-38]:

$$
\operatorname{div}(\overline{\mathbf{f}})=\frac{1}{t_{m}} \int_{0}^{t_{m}} \operatorname{div}(\mathbf{f}) \mathrm{d} t ; \operatorname{div}(\overline{\mathbf{f}})=\sum L_{y}\left(t_{m}\right)
$$

On the other hand, from the simulation results it follows that:

$$
\operatorname{div}(\overline{\mathbf{f}})=-1.0044 ; \sum L_{y}\left(t_{m}\right)=-1.0045
$$

which is in accordance with Eq (42) with a relative error of $4.166 .10^{-4} \%$. Thus it can be concluded that the numerical computations are correct. The power spectral density for the mass position has been plotted in Fig $9 \mathrm{~d}$, in which the strong energy decay can be considered as another indicator of chaos. It should be noticed that Eq (43) is verified when the Lyapunov exponents have reached their steady state value, which in high dimension systems could require long time simulations. Taking into account all the previous results, we can reasonably affirm that the considered electromechanical device has a chaotic dynamic for the parameter values used in Figs 7-8.

Figure 9

\section{Chaotic behavior and how to use it to reach a predetermined set point}

In this section we are going to calculate the Melnikov function to obtain sufficient conditions for chaotic motion as well as to deduce the way of using the chaotic motion to reach a desired set point.

4.1 Chaotic behavior and Melnikov function 
The purpose of this subsection is to investigate the presence of homoclinic orbits and chaotic oscillations. Assuming that $\alpha_{11}>1, \alpha_{z 1}=0, \alpha_{z 2}=0$ and $\alpha_{2} \gg 1$ in accordance with Eq (14), the value of the deviation current $z_{3}{ }_{3}(t)$ will be very small and approximately constant, which will allow to obtain a simplified mathematical model of the device consisting of the following equations:

$$
\begin{aligned}
& \dot{z}_{1}^{\prime}(t)=z_{2}^{\prime}(t) \\
& \dot{z}_{2}^{\prime}(t)=-\sigma^{2}\left[z_{1}^{\prime}(t)+z_{1 e}\right]-\beta\left[z_{1}^{\prime 3}(t)+3 z_{1 e} z_{1}^{\prime 2}(t)+3 z_{1 e}^{2} z_{1}^{\prime}(t)+z_{1 e}^{3}\right]- \\
& -\frac{a_{m}\left[\bar{z}_{3}+i_{0 e}\right]^{2}}{\left[d_{b}+z_{1}^{\prime}(t)+z_{1 e}\right]^{2}}+f_{0}+\left(1-f_{d}\right)\left[z_{1}^{\prime}(t)+z_{1 e}+b\right] A_{d}^{2} \omega_{d}^{2} \sin ^{2} \omega_{d} t
\end{aligned}
$$

If the initial condition $z_{30}^{\prime}=z_{3}^{\prime}(0)$ is a very small value, taking into account that $\mathrm{i}_{0 \mathrm{e}} \approx 0.1-0.2 \mathrm{~A}$ (see table 1 ) it is deduced that $\left(\bar{z}_{3}^{\prime}+i_{0 e}\right)^{2} \approx 2 i_{0 e} \bar{z}_{3}^{\prime}+i_{0 e}^{2}$. From these considerations, assuming that there are no external disturbances and expanding the nonlinear terms of the second Eq. (44) in Taylor series around the origin, it follows that:

$$
\begin{aligned}
& \dot{z}_{1}^{\prime}(t)=z_{2}^{\prime}(t) \\
& \dot{z}_{2}^{\prime}(t)=-p z_{1}^{\prime}(t)-q z_{1}^{\prime 2}(t)-r z_{1}^{\prime 3}(t)
\end{aligned}
$$

where:

$$
\begin{gathered}
p=\sigma^{2}+3 \beta z_{1}^{\prime}(t) z_{1 e}^{2}-\frac{2 a_{m} i_{0 e}\left(2 \bar{z}_{3}+i_{0 e}\right)}{\left(d_{b}+z_{1 e}\right)^{3}} ; q=3 \beta z_{1 e}+\frac{3 a_{m} i_{0 e}\left(2 \bar{z}_{3}+i_{0 e}\right)}{\left(d_{b}+z_{1 e}\right)^{4}} \\
r=\beta-\frac{4 a_{m} i_{0 e}\left(2 \bar{z}_{3}+i_{0 e}\right)}{\left(d_{b}+z_{1 e}\right)^{5}}
\end{gathered}
$$

It should be noticed that there is no damping in the approximate model given by Eqs (45) and (46), so it can be considered as a Hamiltonian system. Consequently, Eqs (45)(46) allows to deduce the parametric equations of the homoclinic orbit [18,19], [25-29], i.e.:

$$
\left[z_{1}^{\prime}(t)\right]_{ \pm}= \pm \frac{2 p_{1}}{C \cosh \left(\sqrt{p_{1}} t\right) \mp q_{1}} ;\left[z_{2}^{\prime}(t)\right]_{ \pm}=\mp \frac{2 p_{1} C \sinh \left(\sqrt{p_{1}} t\right)}{\left(C \cosh \left(\sqrt{p_{1}} t\right) \mp q_{1}\right)^{2}}
$$


where $-\mathrm{p}=\mathrm{p}_{1}>0, \mathrm{q}_{1}=-2 \mathrm{q} / 3>0, \mathrm{r}_{1}=\mathrm{r} / 2$ and $\mathrm{C}^{2}=4 \mathrm{p}_{1} \mathrm{r}_{1}+\mathrm{q}_{1}^{2}$.

Once a homoclinic orbit has been obtained and the corresponding parametric equations have been deduced, it is possible to calculate the Melnikov's function [1819], [25-29]. Taking into account Eqs (44)-(47), the Melnikov function can be written as follows:

$$
\begin{gathered}
M_{p}\left(t_{0}\right)=K \sin 2 \omega_{p} t_{0}\left[2 p_{1} I_{1 p}\left(t_{0}\right)+r_{1 e} I_{2 p}\left(t_{0}\right)\right] ; K=\omega_{p}^{2} A_{p}^{\prime 2} C p_{1} \sqrt{p_{1}}>0 \\
I_{1 p}\left(t_{0}\right)=-\int_{-\infty}^{\infty} \frac{\sinh \left(\sqrt{p_{1}} t\right)}{\left[C \cosh \left(\sqrt{p_{1}} t\right)-q_{1}\right]^{3}} \sin \left(2 \omega_{p} t\right) \mathrm{d} t \\
I_{2 p}\left(t_{0}\right)=-\int_{-\infty}^{\infty} \frac{\sinh \left(\sqrt{p_{1}} t\right)}{\left[C \cosh \left(\sqrt{p_{1}} t\right)-q_{1}\right]^{2}} \sin \left(2 \omega_{p} t\right) \mathrm{d} t \\
M_{n}\left(t_{0}\right)=K \sin 2 \omega_{p} t_{0}\left[2 p_{1} I_{1 n}\left(t_{0}\right)+r_{1 e} I_{2 n}\left(t_{0}\right)\right] ; K=\omega_{p}^{2} A_{p}^{\prime 2} C p_{1} \sqrt{p_{1}}>0 \\
I_{1 n}\left(t_{0}\right)=I_{1 p}\left(t_{0}\right) ; I_{2 n}\left(t_{0}\right)=-I_{2 p}\left(t_{0}\right)
\end{gathered}
$$

where the subscripts " $p$ " and " $n$ " refer to the right (positive) and left (negative) lobes respectively. The integrals of Eqs (48) can be calculated by using standard methods of complex variable [38]. It should be noted that there is a sign change in the functions $\mathrm{M}_{\mathrm{p}}\left(\mathrm{t}_{0}\right)$ and $\mathrm{M}_{\mathrm{n}}\left(\mathrm{t}_{0}\right)$, and thus the sufficient conditions for chaotic behavior are verified.

To analyze the chaotic motion when the transducer platform oscillates harmonically Eqs (12) have been simulated taking $A_{d}=0.275 \mathrm{rad}$ and $\omega_{d}=17.35 \mathrm{rad} / \mathrm{s}$. In Fig 10 the homoclinic orbit has been plotted together with the strange attractor, which seems more dense in comparison with the one shown in fig 9 a. Figs $11 \mathrm{a}$ and b show a clear sensitive dependence. On the other hand, Figs $11 \mathrm{c}$ and $\mathrm{d}$ show the Lyapunov exponents and the power spectral density respectively, for which the same considerations can be made as in the case of chaotic behavior due to a harmonically varying first Lyapunov value.

Figure 10

Figure 11 
4.2 Using the chaotic behavior to reach a predetermined set point

In this subsection we shall use the chaotic behaviors to drive the transducer to a prescribed position, which will be analyzed for the two previously considered routes to obtain chaos. It should be noticed that the control laws defined by Eqs (8)-(10) can be used for both chaos procedures depending on the chosen values for $\alpha_{1}, \alpha_{11}, \alpha_{\mathrm{z} 1}$ and $\alpha_{\mathrm{z} 2}$. According to the discussion of section 3 and subsection 4.1, the system starts with $\alpha_{1}=$ 0 and chaotic oscillations can appear when either $\alpha_{z 1}$ is harmonically varied or an external harmonic disturbance on the platform appears.

Our purpose is to exploit the fact that a chaotic orbit will always be very close to a set point located inside the strange attractor. Consequently, it would be possible to reach the desired set point with small control efforts by varying the control laws given in $\mathrm{Eq}(10)$ and modifying the value of $\mathrm{f}_{0}$ in $\mathrm{Eq}(15)$ so that one stable equilibrium point set point- is obtained. The practical implementation of this issue consists of defining a capture region around the set point $\Omega \equiv\left(\mathrm{r}_{\mathrm{cx}}, \mathrm{r}_{\mathrm{cy}}\right)$ located inside the strange attractor in the phase plane $r_{1}(t)-d r_{1}(t) / d t$ and changing the control law when a chaotic trajectory enters the capture region. For this purpose, we will consider the control laws defined by Eqs (8)-(10) and will assume that the nonlinear control laws are defined by:

For $t<t_{\mathrm{con}}$ :

$$
\left\{\begin{array}{c}
F(t)=F_{0}+K_{p}\left[z_{1}^{\prime}(t)+z_{1 e}\right]+B_{f} z_{2}^{\prime}(t)-m\left[z_{1}^{\prime}(t)+z_{1 e}+b\right] A_{d}^{2} \omega_{d}^{2} \sin ^{2} \omega_{p} t \\
V(t)=R_{g s}\left[z_{3}^{\prime}(t)+i_{0 e}\right]-\frac{2 a z_{2}^{\prime}(t)\left[z_{3}^{\prime}(t)+i_{0 e}\right]}{\left[d_{b}+z_{1 e}+z_{1}^{\prime}(t)\right]}+\left[\frac{2 a}{d_{b}+z_{1 e}+z_{1}^{\prime}(t)}\right] f\left[z_{2}^{\prime}(t), z_{3}^{\prime}(t)\right] \\
f\left[z_{2}^{\prime}(t), z_{3}^{\prime}(t)\right]=-\alpha_{11}^{2} z_{3}^{\prime}(t)-\alpha_{L z 1} \cos \left(\omega_{z} t\right) z_{2}^{\prime 2}(t)-\alpha_{z 2} z_{2}^{\prime 3}(t)-\alpha_{2}^{2} z_{3}^{\prime 3}(t)
\end{array}\right.
$$

For $t \geq t_{\text {con }}$ :

$$
\left\{\begin{array}{c}
F(t)=F_{0 n}+K_{p}\left[z_{1}^{\prime}(t)+z_{1 e n}\right]+B_{f} z_{2}^{\prime}(t)-m\left[z_{1}^{\prime}(t)+z_{1 e n}+b\right] A_{d}^{2} \omega_{d}^{2} \sin ^{2} \omega_{p} t \\
V(t)=R_{g s}\left[z_{3}^{\prime}(t)+i_{0 e n}\right]-\frac{2 a z_{2}^{\prime}(t)\left[z_{3}^{\prime}(t)+i_{0 e n}\right]}{\left[d_{b}+z_{1 e n}+z_{1}^{\prime}(t)\right]}+\left[\frac{2 a}{d_{b}+z_{1 e n}+z_{1}^{\prime}(t)}\right] f\left[z_{2}^{\prime}(t), z_{3}^{\prime}(t)\right] \\
f\left[z_{2}^{\prime}(t), z_{3}^{\prime}(t)\right]=\alpha_{1} z_{2}^{\prime}(t)-\alpha_{11 n}^{2} z_{3}^{\prime}(t)-\alpha_{2}^{2} z_{3}^{\prime 3}(t)
\end{array}\right.
$$

Regarding Eqs (50), the following remarks must be considered: 
- Assuming that there are not external harmonic disturbances on the platform $\left(\mathrm{A}_{d}\right.$ $=0$ ) and taking $\alpha_{\mathrm{Lz} 1} \neq 0$ and $\omega_{\mathrm{z}} \neq 0$, chaotic behavior can arise due to the harmonic variation of the first Lyapunov value. On the other hand, taking $\alpha_{\mathrm{Lz} 1}$ $=0, A_{d} \neq 0$ and $\omega_{d} \neq 0$, the Melnikov theory provides with necessary conditions to obtain chaotic oscillations.

- The value for $F_{0}$ is chosen to obtain three or more equilibrium points as shown in Fig 3 b. So, the harmonic variation of $\alpha_{z 1}\left(\alpha_{z 1}=\alpha_{L z 1} \cos \omega_{z} t\right)$ or the presence of harmonic disturbances on the platform for $t<t_{c o n}$ can give rise to chaotic oscillations (see Figs 5- 8 and Figs 10-12).

- For $t \geq t_{c o n}$ the value for $\mathrm{F}_{0}$ is changed so that $\mathrm{F}_{0 \mathrm{n}}<\mathrm{F}_{0}$, and thus it is possible to choose an equilibrium voltage $\mathrm{V}_{0}$ for which the system has only one equilibrium point. In addition, for an adequate value of $\alpha_{1}$, the eigenvalues of the Jacobian matrix defined by Eq (17) have negative real part and consequently the equilibrium point will be asymptotically stable.

- For $t \geq t_{\text {con }}$, new values $\alpha_{11 \mathrm{n}} \geq \alpha_{11}, \alpha_{\mathrm{Lz} 1}=0$ and $\alpha_{\mathrm{z} 2}=0$ are chosen to remove the Andronov-Poincaré-Hopf bifurcation conditions or the chaos conditions deduced from Melnikov theory, so that the control laws can drive the transducer to the desired position.

The previous ideas have been applied to obtain Fig 12 taking $\mathrm{d}=0.04 \mathrm{~m}$ and $\mathrm{F}_{0}$ $=7.1343 \mathrm{~N}$ to obtain three equilibrium points. The chosen set point $\mathrm{r}_{1 \mathrm{e}}=0.07\left(\mathrm{z}_{1 \mathrm{e}}=\mathrm{r}_{1 \mathrm{e}}-\right.$ b) corresponds to an equilibrium current $\mathrm{i}_{0 \mathrm{e}}=0.1827 \mathrm{~A}$ and to an equilibrium voltage $\mathrm{V}_{0 \mathrm{e}}=221.0318 \mathrm{~V}$. Figs $12 \mathrm{a}$ and $\mathrm{b}$ show the sensitive dependence for two simulations with very close initial conditions for $\mathrm{V}_{0}=227.2167 \mathrm{~V}\left(\mathrm{i}_{0 \mathrm{e}}=0.1878 \mathrm{~A}\right), \mathrm{F}_{0}=7.1343 \mathrm{~N}$, $\mathrm{A}_{\mathrm{d}}=0, \omega_{\mathrm{d}}=0, \alpha_{1}=0, \alpha_{2}=10, \alpha_{11}=1, \alpha_{\mathrm{Lz} 1}=0.05, \alpha_{\mathrm{z} 2}=0.001, \omega_{\mathrm{z}}=0.995 \mathrm{rad} / \mathrm{s}$ and the equilibrium point $r_{1 e}=0.07$ with eigenvalues $[ \pm 21.699,-1]$. It should be noticed that the equilibrium point $\left(r_{1 e n} \equiv r_{1 e}=0.07\right)$ has been reached when a capture region has been defined by $r_{c x}=0.02 \mathrm{~m}, r_{c y}=0.1 \mathrm{~m} / \mathrm{s}$ and the control law has been changed at $\mathrm{t}=\mathrm{t}_{\mathrm{con}}=$ 160, in accordance with Eqs (60). In Figs $12 \mathrm{c}$ and $\mathrm{d}$ the control signals have been plotted to confirm their physical feasibility. The fourth order Runge-Kutta integration method has been used taking initial conditions zpo1 $=\left[0.005,0,10^{-4}, 0,0\right]$, a simulation time $\mathrm{t}_{\mathrm{m}}=200 \mathrm{~s}$, and a simulation step $\mathrm{T}=2 \cdot 10^{-3}$. 
With the purpose of validating the robustness of the nonlinear control laws given by Eqs (50), we consider that the deviation variables are affected by a random noise, for which we will assume that:

$$
\begin{aligned}
& z_{1}^{\prime}(t)=z_{1}^{\prime}(t)+f_{n a}[X-0.5] \\
& z_{2}^{\prime}(t)=z_{2}^{\prime}(t)+f_{n a}[X-0.5] \\
& z_{3}^{\prime}(t)=z_{3}^{\prime}(t)+f_{n a}[X-0.5]
\end{aligned}
$$

where $X$ is a random variable that is uniformly distributed between 0 and 1 , and $\mathrm{f}_{\text {na }}>0$ is an amplification factor to obtain a uniformly distributed noise amplitude between $\mathrm{f}_{\text {na }} / 2$ and $\mathrm{f}_{\text {na }} / 2$ [23]. Taking into account the control law given by Eqs (50), two simulations of Eqs (12) are plotted in Fig 13. On one hand, in fig $13 \mathrm{a}$ it is assumed that there is no noise, and the desired set point (which coincides with the initial conditions) is reached when a chaotic trajectory enters in the capture zone. On the other hand fig 13 $b$ shows the simulation results assuming a random noise with $f_{n a}=0.04$, which is obviously higher than the typical noise level present in a standard measurement equipment. In this case a steady state error appears.

\section{Figure 13}

To overcome this problem, a linear PI controller has been added to the control force, which is now defined as:

$$
\begin{gathered}
F(t)=F_{0}+K_{p}\left[z_{1}^{\prime}(t)+z_{1 e}\right]+B_{f} z_{2}^{\prime}(t)-f_{d} m\left[z_{1}^{\prime}(t)+z_{1 e}+b\right] A_{p}^{2} \omega_{p}^{2} \sin ^{2} \omega_{p} t- \\
-m C_{p}\left[z_{1}^{\prime}(t)+\frac{1}{\tau_{i}} \int_{0}^{t} z_{1}^{\prime}(\tau) \mathrm{d} \tau\right]
\end{gathered}
$$

where $C_{p}$ is the proportional action constant and $\tau_{i}$ is the integral action or reset time [32-34]. Although the equilibrium points remain unchanged, the integral action gives rise to a system of four state variables. Assuming that there is no random noise, substituting Eq (52) into Eqs (7) and taking into account Eqs (6), (11) and (12), the linear part of the system can be written as follows: 


$$
\left[\begin{array}{c}
\dot{z}_{1}^{\prime}(t) \\
\dot{z}_{2}^{\prime}(t) \\
\dot{z}_{3}^{\prime}(t) \\
\dot{I}(t)
\end{array}\right]=\left[\begin{array}{cccc}
0 & 1 & 0 & 0 \\
-a_{21}-C_{p} & 0 & -a_{23} & -C_{p} / \tau_{i} \\
0 & \alpha_{1} & -\alpha_{11}^{2} & 0 \\
1 & 0 & 0 & 0
\end{array}\right]\left[\begin{array}{c}
z_{1}^{\prime}(t) \\
z_{2}^{\prime}(t) \\
z_{3}^{\prime}(t) \\
I(t)
\end{array}\right] ; I(t)=\int_{0}^{t} z_{1}^{\prime}(\tau) \mathrm{d} \tau
$$

From $\mathrm{Eq}(53)$ the $\mathrm{C}_{\mathrm{p}}$ and $\tau_{\mathrm{i}}$ values can be chosen so that all the eigenvalues of the matrix defined in $\mathrm{Eq}$ (53) have negative real part, and consequently the set point will be an asymptotic equilibrium point. On the other hand, in the presence of noise the equilibrium point will be reached with a small oscillation around it.

To corroborate the previous reasoning, Eqs (12) have been simulated assuming that there is no harmonic disturbance in the platform and that the conditions of the Andronov-Poincaré-Hopf bifurcation are verified. Fig 14 a shows that the system is chaotic for $\mathrm{t}<\mathrm{t}_{\text {con }}$. At $\mathrm{t}=\mathrm{t}_{\text {con }}=70 \mathrm{~s}$, the control laws are changed in accordance with Eqs (50) and Eq (52). The chosen values for the PI control action $\left(C_{p}=1 \mathrm{~s}^{-1}, C_{p} / \tau_{i}=15\right.$ $\mathrm{s}^{-2}$ ) give rise to an asymptotically stable desired set point. It should be noticed that the random noise makes the transducer to remain with small oscillations around the set point with a mean value of 0.07 , as it clearly appears in Fig 14 b.

\section{Figure 14}

It is important to remark that the integral action $I(t)$ given in Eq (53) should not reach too high values as it may prevent the system to reach the set point. Consequently, it may be necessary to limit the values of $|I(t)|$ to certain upper bound that must not be exceeded. As an example, Fig 15 shows two cases in which the chaotic motion is due to external harmonic disturbances on the platform with random noise, where the integral action $I(t)$ has been limited to a maximum absolute value of 0.11 . From the simulation results it can be deduced that the set point is reached for $C_{p}=0.5 \mathrm{~s}^{-2}$ and $\tau_{\mathrm{i}}=0.1428 \mathrm{~s}^{-1}$; however for small and high values of $C_{p}$ and $\tau_{i}$ respectively $\left(C_{p}=10^{-6} \mathrm{~s}^{-2}, \tau_{i}=1 \mathrm{~s}^{-1}\right)$ it may be necessary to increase the corresponding values $r_{c x}$ and $r_{c y}$ of the capture zone so that the set point can be reached. The control is applied at $t_{c o n}=65 \mathrm{~s}$ taking $\mathrm{F}_{0}=0.5 \mathrm{~N}$, $\alpha_{1}=20, \alpha_{2}=10, \alpha_{11}=5, \alpha_{z 1}=0, \alpha_{z 2}=0$ and $\omega_{z}=0$. The eingenvalues of the 
equilibrium point are $[-11.8344 \pm 48.5334 \mathrm{j},-0.0269,-1.3043]$ when the PI controller is present and [-11.8302 $\left.\pm 48.5293 \mathrm{j},-10^{-5},-1.3253\right]$ when PI controller is not included.

Figure 15

Other control strategies such as the control partial technique [35] represent an alternative procedure for using the chaotic behavior to maintain the system in a small zone of the phase plane employing very small control signals. In this sense, the sensitive dependence has been applied in several contexts, for which details can be found in Refs [39-40].

\section{Conclusions}

In this paper the chaotic oscillations of an electromechanical transducer have been examined. The device is controlled by a control voltage $\mathrm{V}(\mathrm{t})$ and an external control force $\mathrm{F}(\mathrm{t})$, which can be chosen with the classical structure of the PD control plus certain nonlinear terms. An admissible range of parameter values for the proposed control laws has been deduced, which has allowed to drive the transducer mobile mass to any desired equilibrium point.

The stability analysis of the weak focuses associated to this bifurcation has been carried out from the analytic calculation of the first Lyapunov value. It has been both analytically and numerically corroborated that a weak focus is stable or unstable if its corresponding first Lyapunov value is negative or positive respectively. This property has been used to induce chaotic motions by means of a harmonic variation of the first Lyapunov value, whose sign change gives rise to an interchange of stability between the two weak focuses.

From a simplified model of the controlled system, the Melnikov theory has been be applied to obtain sufficient conditions for chaos when the platform is harmonically varied, which provides another route to obtain chaos. The chaotic oscillations have been corroborated by calculating the sensitive dependence, Lyapunov exponents and the power spectral density. 
Another interesting aspect of this paper is the possibility of using chaotic oscillations to drive the transducer mass to a prescribed set point, even in the presence of random noise associated to the measurement process. For this purpose, a capture region around the set point is defined, which allows to change the control laws to reach the (stable) equilibrium point when a chaotic trajectory enters the capture region. The presence of random noise gives rise to a steady state error, which is removed by adding a linear PI controller to the control force.

The simulation process has been carried out by using the Runge-Kutta integration method of order four with simulation steps of $10^{-4} \mathrm{~s}$ introducing the artificial variable $\mathrm{z}_{4}{ }^{\prime}(\mathrm{t})$ to obtain an autonomous system (Eqs (12) and (41)). On the other hand, the Lyapunov exponents have been calculated inside the main Runge-Kutta loop by means of the Runge-Kutta-Fehlberg method to avoid problems of numerical instability. The control laws given by Eqs (8) and (9) are changed through Eqs (50)-(53) by adding random noise given by Eq (51), which is incorporated through random variables in the computational simulation.

As a concluding remark, this paper shows a framework in which the nonlinear model of an electromechanical transducer, the design of a nonlinear control system in presence of random noise, self-oscillations originated by an Andronov-Poincaré-Hopf bifurcation, Melnikov theory and chaotic oscillations are presented in a unified viewpoint. The methodology of these investigations can be extended to more complicated nonlinear dynamical systems with more complicated behaviors, such as double Hopf bifurcations or Bogdanov-Takens bifurcations.

\section{Acknowledgments}

This work was supported by the "Generalitat Valenciana" (Spain) under project GV/2012/099 and by "Ministerio de Ciencia e Innovación" (Spain) under project FIS2011-29803-C02-01. 


\section{References}

[1] A.A. Andronov, A.A. Vitt, S.E. Khaikin, Theory of Nonlinear Oscillations, Dover, New York, 1966.

[2] J. Meissel, Principles of Electromechanical Energy Conversion, McGraw-Hill, New York, 1966.

[3] J. Awrejcewicz and Z. Koruba, Classical Mechanics. Applied Mechanics and Mechatronics, Springer, New York, 2012.

[4] R. Yamapi Dynamics of an electromechanical damping device with magnetic coupling. Communication in Nonlinear Sciences and Numerical Simulation, 11, (2006) 907-921.

[5] M.F. Pérez-Polo, M.Pérez-Molina, Fold-Hopf bifurcation, steady state, selfoscillating and chaotic behavior in an electromechanical transducer with nonlinear control, Communications in Nonlinear Sciences and Numerical Simulations, 17 (2012) 129-152.

[6] Chang Shun-Chang, Lin Hai-ping, Non-Linear dynamics and Chaos control for an electromagnetic system, Journal of Sound and Vibration, 279 (2005) 327-344.

[7] R. Yamapi, M.A. Aziz-Alaoui. Vibration analysis and bifurcations in the selfsustained electromechanical system with multiple functions. Communications in Nonlinear Science and Numerical Simulation, 12 (2007) 1534-1549.

[8] R. Yamapi, J. B. Chabi Orou, P. Woafo, Harmonic oscillations, stability and chaos control in a non-linear electromechanical system, Journal of Sound and Vibration, 259 (5) (2003) 1253-1264.

[9] J.C. Ji, Nonresonant Hopf bifurcations of a controlled van der Pol-Duffing oscillator, Journal of Sound and Vibration, 297 (2006) 183-199.

[10] Ahmad M. Harb, Nonlinear chaos control in a permanent magnet reluctance machine. Chaos Solitons \& Fractals, 19 (2004) 1227-1224.

[11] F.M. Moukam Kakmeni, S. Bowong, C. Tchawoua, E. Kaptouom, Strange attractors and chaos control in a Duffing-Van der Pol oscillator with two external periodic forces, Journal of Sound and Vibration, 277 (2004) 783-799.

[12] M. Siewe Siewe, S.B. Yamgoué, F.M. Moukam Kakmeni, C. Tchawoua, Chaos controlling self-sustained electromechanical seismograph system based on the Melnikov theory. Nonlinear Dynamics, 62 (2011) 379-389.

[13] R. Shabani, S. Tariverdilo, G. Rezazadeh, A.P. Agdam, Nonlinear vibrations and chaos in electrostatic torsional actuators, Nonlinear Analysis Real Word Applications, 12 (2011) 3572-3584.

[14] S. Haghighi Hossein, H.D. Markazi Amir, Chaos prediction and control in MEMS resonators. Communications in Nonlinear Science and Numerical Simulation, 15 (2010) 3091-3199.

[15] S. Liu, A. Davidson, Q, Lin, Simulation studies on nonlinear dynamics and chaos in a MEMS cantilever control system, Journal of Micromechanics and Microengineering, 14 (2004) 1064-1073.

[16] L. Qunhong, X. Jiezhen, H. Cuncai, Bifurcations of a micro-electromechanical nonlinear coupling system. Communication in Nonlinear Science and Numerical Simulation, 16 (2011) 769-775.

[17] C.A. Kitio Kwuimy, B.Nana, P.Woafo, Experimental bifurcations and chaos in a modified self-sustained macro electromechanical system. Journal of Sound and Vibration, 329 (2010) 3137-3148.

[18] J. Guckenheimer, P. Holmes, Nonlinear Oscillations, Dynamical Systems and Bifurcations of Vector Fields, Springer, New York 1983. 
[19] S. Wiggins, Introduction to Applied Nonlinear Dynamical Systems and Chaos, second ed., Springer, New York 2000.

[20] Y. A. Kuznetsov, Elements of Applied Bifurcation Theory, third ed., Springer, New York 2004.

[21] D. Wang, R. Mao, Improved K-K algorithm for computing Lyapunov values. Tatra Mountains Mathematical Publications, 4 (1994) 235-242.

[22] A. L. Shil'nikov, Bifurcation and chaos in the Marioka-Sumizu System, Selecta Mathematica Sovietica (Birkäuser Verlag) 10 (1991) 105-117.

[23] L.P. Shilnikov, A.L. Shilnikov, D.V. Turaev, L.O. Chua, Methods of Qualitative Theory in Nonlinear Dynamics. Part II, World Scientific, New Jersey, 2001.

[24] P.Glendinning, Stability, Instability, and Chaos: An Introduction to the Theory of Nonlinear Differential Equations. Cambridge Univ. Press, Cambridge 1994.

[25] J. Awrejcewicz, M.M. Holicke, Smooth and Nonsmooth High Dimensional Chaos and the Melnikov-Type Methods. World Scientific Publishing, Singapore, 2007

[26] Zhouchao Wei, Qigui Yang, Dynamical analysis of a new autonomous 3-D chaotic system only with stable equilibria, Nonlinear Analylsis Real Word Applications, 12 (2011) 106-118.

[27] M. Siewe Siewe, C. Tchawoua, P. Woafo, Melnikov chaos in a periodically driven Rayleigh-Duffing oscillator, Mechanics Research Communications, 37 (2010) 363-368.

[28] M.F. Perez-Polo, M. Perez-Molina, J.Gil Chica, Swing-up and positioning control of an inverted wheeled cart pendulum system with chaotic balancing motions. International Journal of Non-Linear Mechanics, 47 (2012) 655-665.

[29] L. Ruihong, X. Wei, L. Shuang, Chaos controlling of extended nonlinear Lie'nard system based on the Melnikov theory, Applied Mathematics and Computation, 178 (2006) 405-414.

[30] A.J. Lichtenberg, M.A. Lieberman, Regular and chaotic dynamics, Springer, New York 1992.

[31] K. Stane, P. Matjaz, M. Marko. Detecting chaos from a time series, European Journal of Physics, 26, (2005) 205-215.

[32] J. Ackermann, Sampled-Data Control Systems. Analysis and Synthesis, Robust System Design, Springer_Verlag, Berlin 1985.

[33] K. Ogata, Modern Control Engineering, Prentice-Hall, New York (2000)

[34] P. Albertos, A. Sala, A, Multivariable Control Systems, Springer, London 2004.

[35] A. Wagemakers, S. Zambrano, M. A. Sanjuán, Partial control of transient chaos in electronic circuits, International Journal Bifurcations and Chaos, 22 (2012) 1250032.

[36] G. Benettin, L. Galgani, A. Giorgilly, J.M. Strelcyn, Lyapunov characteristic exponents for smooth dynamical systems and for Hamiltonian systems: a method for computing all of them, part I: theory, Meccanica 15 (1980) 9-20.

[37] G. Benettin, L. Galgani, A. Giorgilly, J.M. Strelcyn, Lyapunov characteristic exponents for smooth dynamical systems and for Hamiltonian systems: a method for computing all of them, part II: numerical applications, Meccanica 15 (1980) 21-30.

[38] L. Perko, Differential Equations and Dynamical Systems, Springer, third ed., New York, 2007.

[39] E. Ott, Choas in Dynamical Systems, Cambridge University Press, second ed., Cambridge, 2002.

[40] E. Ott, C. Gregobi, J.A. Jorke, Controlling Chaos, Phys. Rev. Lett., 64 (1990) 1196-1198. 


\section{Figure captions}

Figure 1. Electromechanical transducer formed by an electric circuit, magnetic coupling, a mobile piece with linear damping, a nonlinear spring and (optional) harmonic platform disturbances. The control signals are $\mathrm{V}(\mathrm{t})$ and $\mathrm{F}(\mathrm{t})$.

Figure 2. Curves for $f\left(r_{1 e}\right)$ as a function of the equilibrium points $r_{1 e}\left(E q(21)\right.$ ) taking $V_{0}$ as a parameter and $\mathrm{F}_{0}=7.1343 \mathrm{~N}$. One, three or four reachable equilibrium points can appear depending on the value for $\mathrm{V}_{0}$.

Figure 3. a) Angular frequencies $\omega$ (taking $\alpha_{1}=0$ in Eq (25)) as a function of the equilibrium points $r_{1 \mathrm{e}}$ which allow to obtain a Andronov-Poincaré-Hopf bifurcation. $b$ ) Curve for the equilibrium points deduced from Fig 2 taking $\mathrm{V}_{0}=227.2167 \mathrm{~V}\left(\mathrm{i}_{0 \mathrm{e}}=\right.$ $0.1878 \mathrm{~A}$ ) and $\mathrm{F}_{0}=7.1343 \mathrm{~N}$. The equilibrium points are $\mathrm{P}_{1}(0.111), \mathrm{P}_{2}(0.07)$-unstable saddle- and $P_{3}(0.0403)$, whose eingenvalues are $\left( \pm 34.9976 \mathrm{j},-\alpha_{11}{ }^{2}\right),\left( \pm 216980,-\alpha_{11}{ }^{2}\right)$ and $\left( \pm 26.3730 \mathrm{j},-\alpha_{11}^{2}\right)$ respectively.

Figure 4. a) First Lyapunov value as a function of the equilibrium points $r_{1 e}$ and the control parameter $\alpha_{z 1}$ assuming that $\alpha_{11}=1, \alpha_{z 2}=0.001$ and that the values for $r_{1 \mathrm{e}}$ are near the equilibrium point $\mathrm{P}_{3}$ (see Fig 3 a)). b) Same parameters as in the previous case but now assuming that the values for $\mathrm{r}_{1 \mathrm{e}}$ are near the equilibrium point $\mathrm{P}_{1}$.

Figure 5. a) Phase plane $r_{1 \mathrm{e}}(\mathrm{t})-\mathrm{dr}_{1 \mathrm{e}}(\mathrm{t})$ taking $\alpha_{1}=0, \alpha_{2}=0, \alpha_{11}=1, \alpha_{\mathrm{z} 1}=-2, \alpha_{\mathrm{z} 2}=0.02$, $\mathrm{r}_{1 \mathrm{e}}=0.07, \mathrm{i}_{0 \mathrm{e}}=0.1878 \mathrm{~A}$ and $\mathrm{V}_{0 \mathrm{e}}=227.2167 \mathrm{~V}$. The equilibrium points are $\mathrm{P}_{1}$ $\left(r_{1 \mathrm{e}}=0.111\right.$, unstable $)$ and $\mathrm{P}_{3}\left(\mathrm{r}_{1 \mathrm{e}}=0.0403\right.$, stable $)$. b) Phase plane $\mathrm{r}_{1 \mathrm{e}}(\mathrm{t})-\mathrm{dr}_{1 \mathrm{e}}(\mathrm{t})$ taking $\alpha_{1}=$ $0, \alpha_{2}=0, \alpha_{11}=1, \alpha_{\mathrm{z} 1}=-1, \alpha_{\mathrm{z} 2}=0.3, \mathrm{r}_{1 \mathrm{e}}=0.07, \mathrm{i}_{0 \mathrm{e}}=0.1878 \mathrm{~A}$ and $\mathrm{V}_{0 \mathrm{e}}=227.2167 \mathrm{~V}$. The equilibrium points are $\mathrm{P}_{1}\left(\mathrm{r}_{1 \mathrm{e}}=0.111\right.$, unstable $)$ and $\mathrm{P}_{3}\left(\mathrm{r}_{1 \mathrm{e}}=0.0403\right.$, unstable $)$.

Figure 6. a) Harmonic variation of the first Lyapunov values (see Fig 4) taking $\alpha_{1}=0$, $\alpha_{2}=10, \alpha_{11}=1, \alpha_{\mathrm{z} 1}=0.05, \alpha_{\mathrm{z} 2}=0.001, \mathrm{r}_{1 \mathrm{e}}=0.07$ and $\omega_{\mathrm{z}}=0.995 \mathrm{rad} / \mathrm{s}$. For $0<\mathrm{t}<\mathrm{t}_{1}$ the first Lyapunov values are $\mathrm{L}_{1}\left(\mathrm{P}_{1}\right)<0-\mathrm{P}_{1}$ and $\mathrm{L}_{1}\left(\mathrm{P}_{3}\right)>0-\mathrm{P}_{3}$. b) Simulation results of Eqs (12) for $\mathrm{f}_{\mathrm{d}}=1$ and the control parameters indicated in the caption of Fig $5 \mathrm{a}$ ).

Figure 7. Simulation results obtained with parameters of the caption of Fig 6. a) Magnetic field in the air gap of the device (see Fig 1). b) Velocity of the mobile mass. c) Solenoid current $\mathrm{i}(\mathrm{t})$, which is almost constant with small variations around the equilibrium current $\mathrm{i}_{0 \mathrm{e}}$. d) The time derivative di(t)/dt of the solenoid current takes very small values.

Figure 8. Simulation results of Eqs (12) assuming $\mathrm{V}_{0}=227.2167 \mathrm{~V}\left(\mathrm{i}_{0 \mathrm{e}}=0.1878 \mathrm{~A}\right), \mathrm{F}_{0}$ $=7.1343 \mathrm{~N} . \mathrm{A}_{\mathrm{d}}=0, \omega_{\mathrm{d}}=0, \alpha_{1}=0, \alpha_{2}=10, \alpha_{11}=1, \alpha_{\mathrm{z} 1}=0.05, \alpha_{\mathrm{z} 2}=0.001, \omega_{\mathrm{z}}=0.995$ $\mathrm{rad} / \mathrm{s}$ and $r_{1 e}=0.07$. a) Sensitive dependence of the mobile mass position $z_{1}^{\prime}(t)=r_{1}(t)-$ $\mathrm{r}_{1 \mathrm{e}}$. b) Error variation as a function of the time for two very close initial conditions. c) Control force given by Eq (8). d) Control voltage given by Eqs (9) and (10). 
Figure 9. a) Strange attractor in the phase plane $z_{1}(t)-z_{2}(t)$ indicating that a weak focus can appear at points $\mathrm{P}_{1}$ or $\mathrm{P}_{3}$. b) Variation with respect to time of the all Lyapunov exponents of the system. c) Variation with respect to time of the Lyapunov exponent sum and the vector field divergence of the system given by Eqs (43). d) Power spectral density for the mobile mass position (the parameter values and simulation data are indicated at the caption of Fig 8).

Figure 10. Strange attractor and homoclinic orbit in terms of deviation variables assuming the following parameter values: $V_{0}=227.2167 \mathrm{~V}\left(\mathrm{i}_{0 \mathrm{e}}=0.1878 \mathrm{~A}\right), \mathrm{F}_{0}=$ 7.1343 N. $A_{d}=0.275, \omega_{d}=17.35 \mathrm{rad} / \mathrm{s}, \alpha_{1}=0, \alpha_{2}=10, \alpha_{11}=5, \alpha_{\mathrm{z} 1}=0, \alpha_{\mathrm{z} 2}=0, \omega_{\mathrm{z}}=0$, $\mathrm{r}_{1 \mathrm{e}}=0.07, \mathrm{p}_{1}=4.7084 .10^{2}, \mathrm{q}_{1}=3.9469 .10^{3}, \mathrm{r}_{1}=1.9497 .10^{5}$ and $\mathrm{C}=1.9565 .10^{4}$.

Figure 11. Simulation results of Eqs (12) for the parameter values indicated in the caption of Fig 10. a) State variables $z_{1}(t), z_{2}(t)$ and $z_{3}(t)$ as a function of the time. b) Sensitive dependence of the mobile mass position obtained for two initial conditions differing in $10^{-10}$. c) Lyapunov exponents of the system as a function of the time. d) Power spectral density for the mobile mass position.

Figure 12. Simulation results of Eqs (12) before applying the control law. The control is applied at $\mathrm{t}_{\mathrm{con}}=160 \mathrm{~s}$ taking $\mathrm{F}_{0 \mathrm{n}}=0.6 \mathrm{~N}, \alpha_{1}=10, \alpha_{2}=10, \alpha_{11 \mathrm{n}}=1, \alpha_{\mathrm{z} 1}=0, \alpha_{\mathrm{z} 2}=0, \omega_{\mathrm{z}}$ $=0$. The equilibrium point is $r_{1 \mathrm{en}}=0.07$ and has eigenvalues $[-0.0877,-0.4561 \pm$ 37.4682j]. a) Sensitive dependence before the control is applied b) Error signal as a function of the time. c) Control force before and after applying the control law. d) Control voltage before and after applying the control law.

Figure 13. Simulation results of Eqs (12) before applying the control law (parameter are indicated in the caption of Fig 12). Simulation time and step are $t_{\mathrm{m}}=100 \mathrm{~s}$ and $\mathrm{T}=$ $5.10^{-4}$ respectively. Control law is applied at $\mathrm{t}_{\mathrm{con}}=70 \mathrm{~s}$ taking $\mathrm{F}_{0}=0.5 \mathrm{~N}, \alpha_{1}=20, \alpha_{2}=$ $10, \alpha_{11}=5, \alpha_{z 1}=0, \alpha_{z 2}=0, \omega_{z}=0$. a) Trajectory without noise after the control is applied, with $r_{c x}=0.02 \mathrm{~m}$ and $r_{c y}=0.1 \mathrm{~m} / \mathrm{s}$. b) Trajectory assuming random noise with amplitude $\mathrm{f}_{\mathrm{na}}=0.04$ after the control is applied (same capture zone).

Figure 14. a) Simulation of the case indicated in Fig $13 \mathrm{~b}$ ) including an integral action with parameters $C_{p}=1 \mathrm{~s}^{-1}$ and $C_{p} / \tau_{i}=15 \mathrm{~s}^{-2}$, which allows to drive the system to the set point (steady-state error is removed). b) Time evolution of the transducer position with and without random noise.

Figure 15. Simulation results of Eqs (12) with a PI controller of limited integral action $\left(\mathrm{C}_{\mathrm{p}}=0.5 \mathrm{~s}^{-1}, \mathrm{C}_{\mathrm{p}} / \tau_{\mathrm{i}}=3.5 \mathrm{~s}^{-2}\right.$ and maximum absolute value 0.11 for the integral action $)$ and without PI controller $\left(\mathrm{C}_{\mathrm{p}}=10^{-6} \mathrm{~s}^{-1}, \mathrm{C}_{\mathrm{p}} / \tau_{\mathrm{i}}=10^{-6} \mathrm{~s}^{-2}\right)$. The equilibrium point $\mathrm{r}_{1 \mathrm{e}}=0.07$ has eigenvalues $[-1.3253,-11.8374 \pm 48.5293 \mathrm{j}]$. Trajectories after applying the control with $\mathrm{f}_{\mathrm{na}}=0.01$ with $\mathrm{r}_{\mathrm{cx}}=0.04 \mathrm{~m}, \mathrm{r}_{\mathrm{cy}}=0.1 \mathrm{~m} / \mathrm{s}$. 


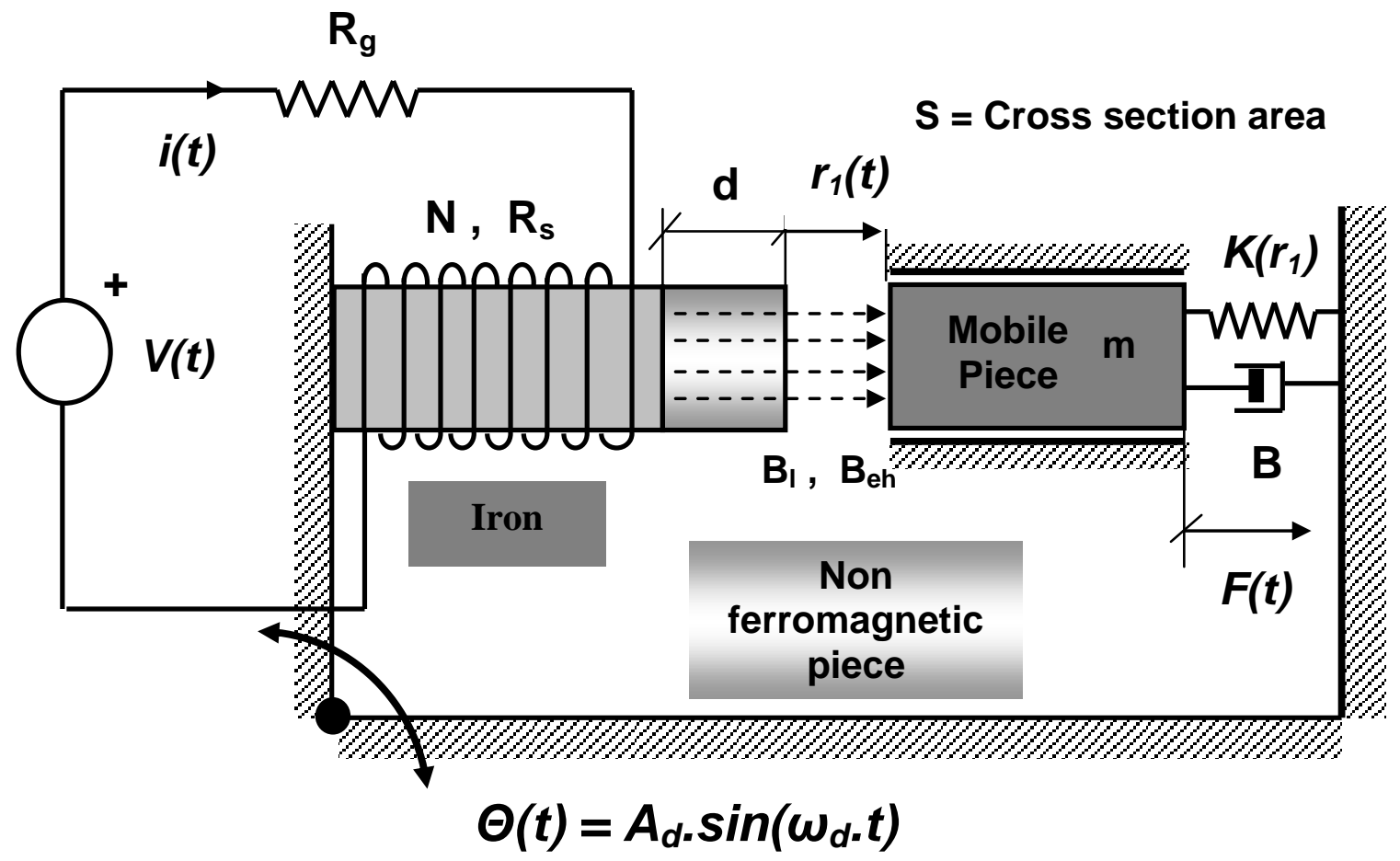

nelec_office_bw_Fig1 


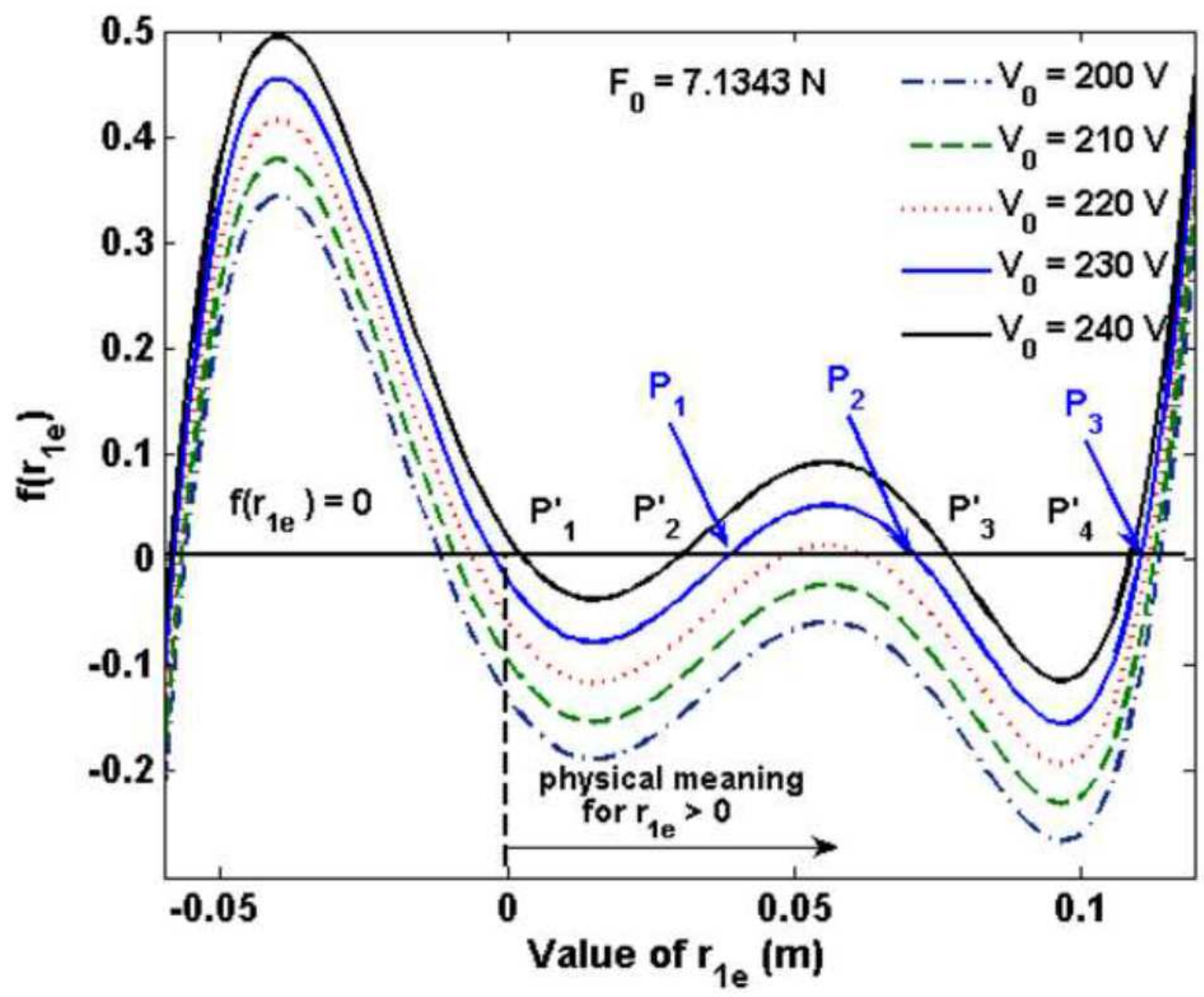



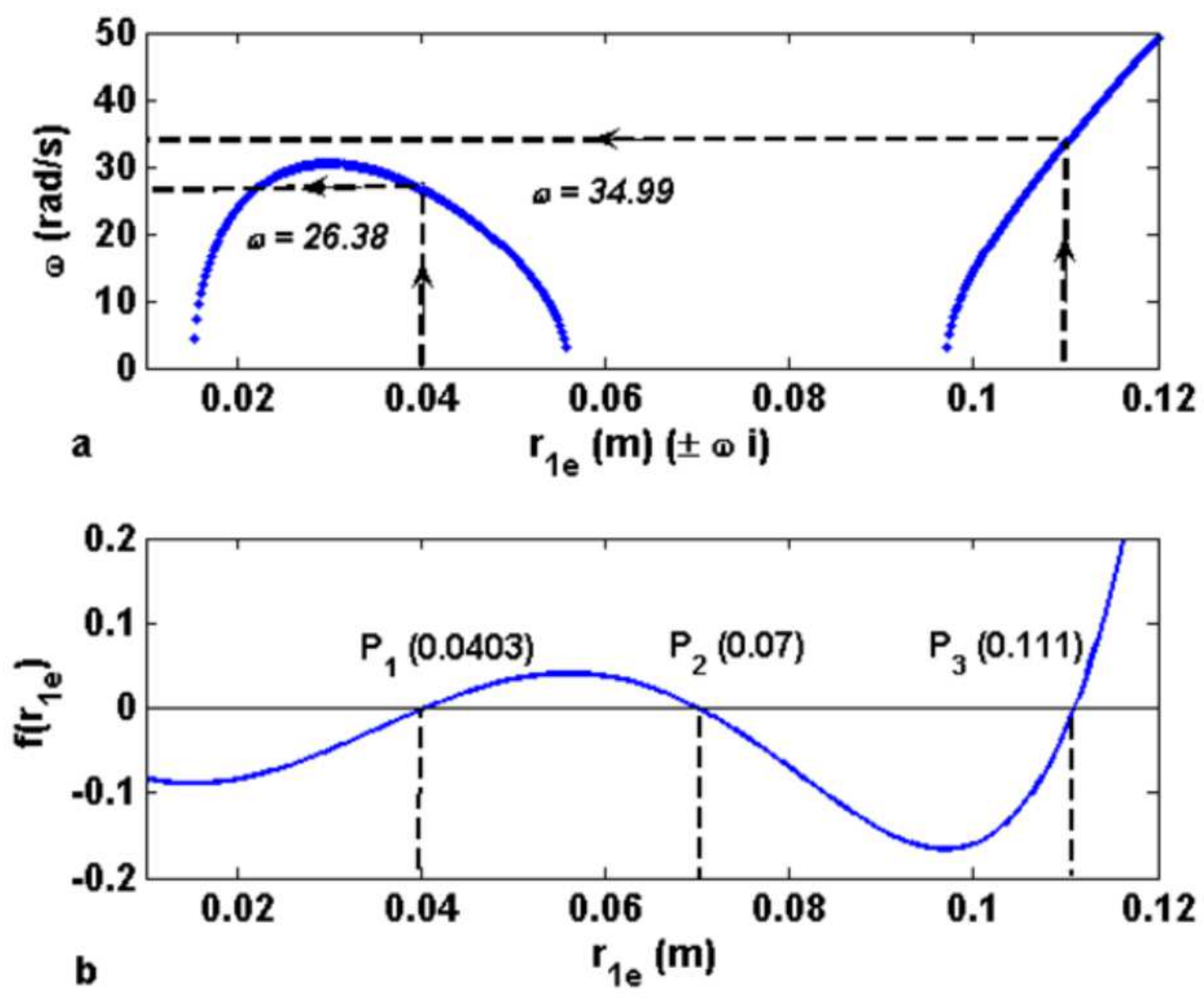

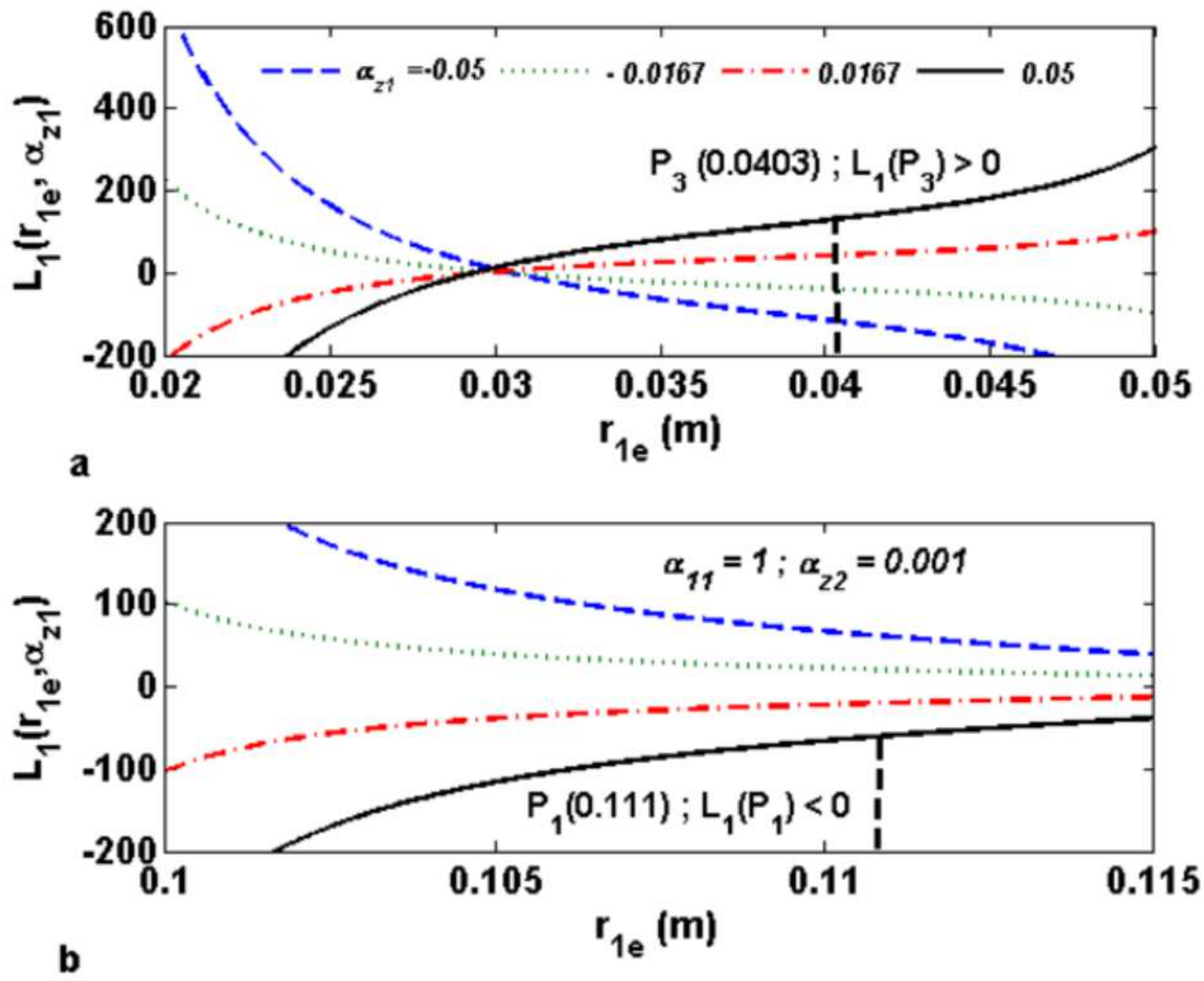

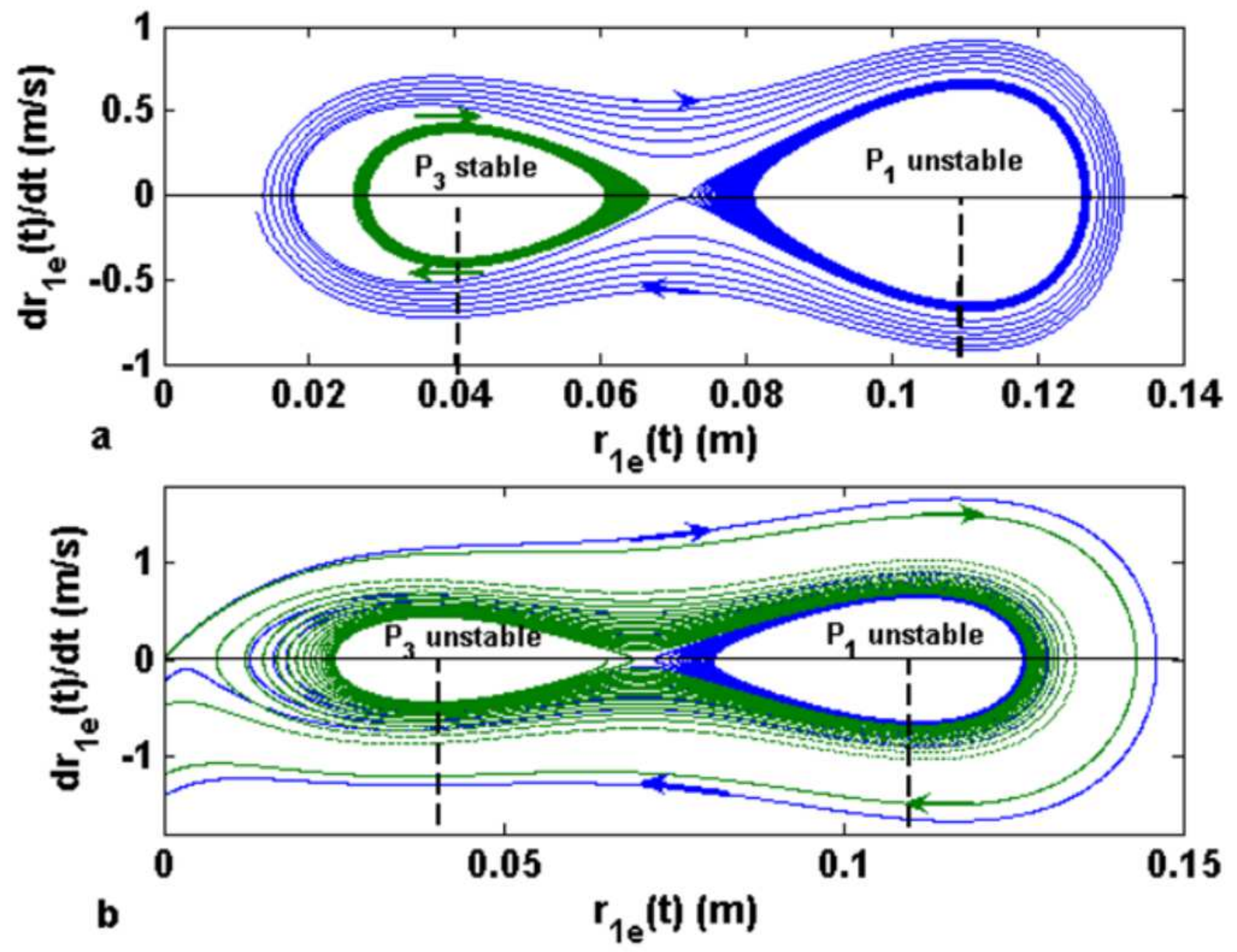

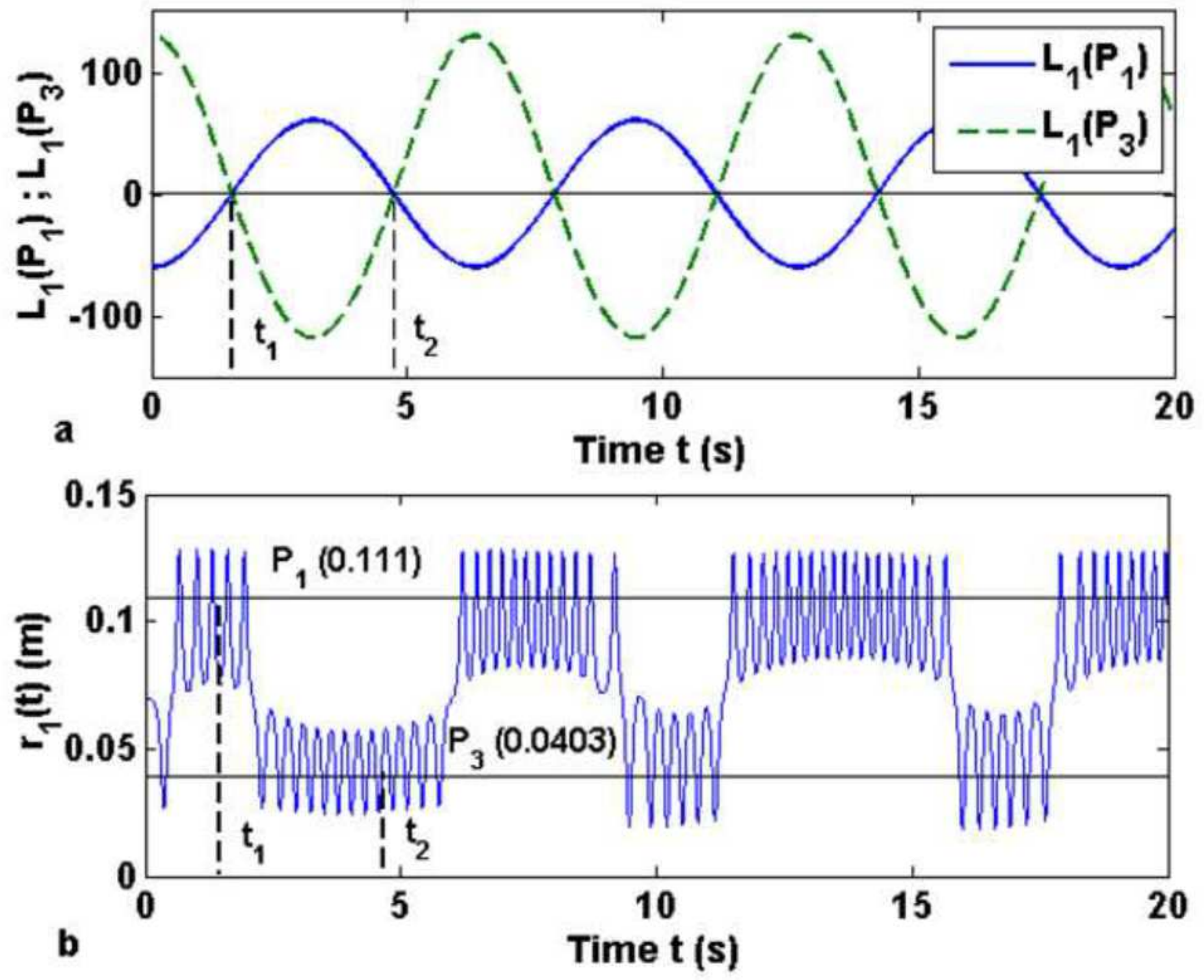

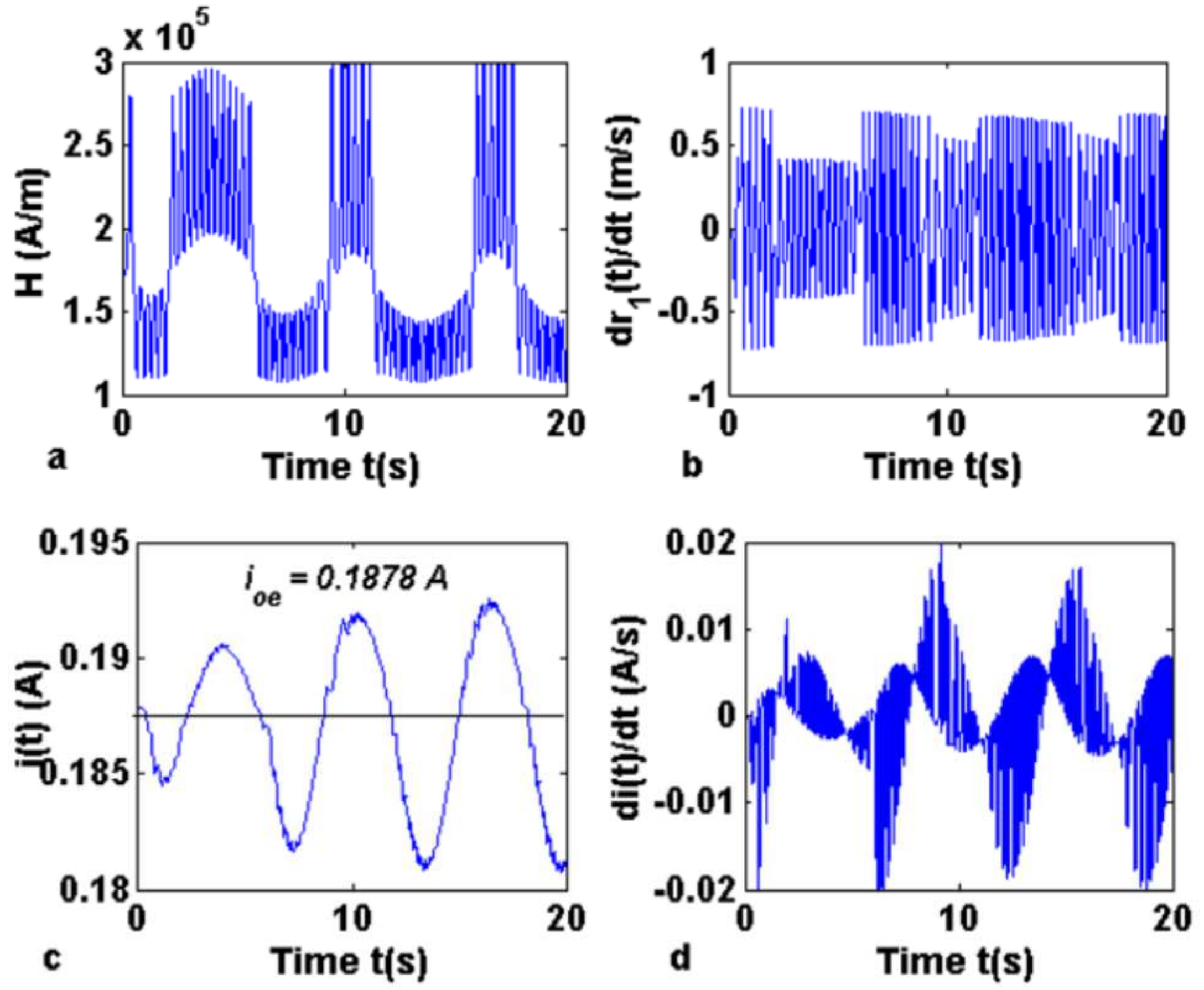

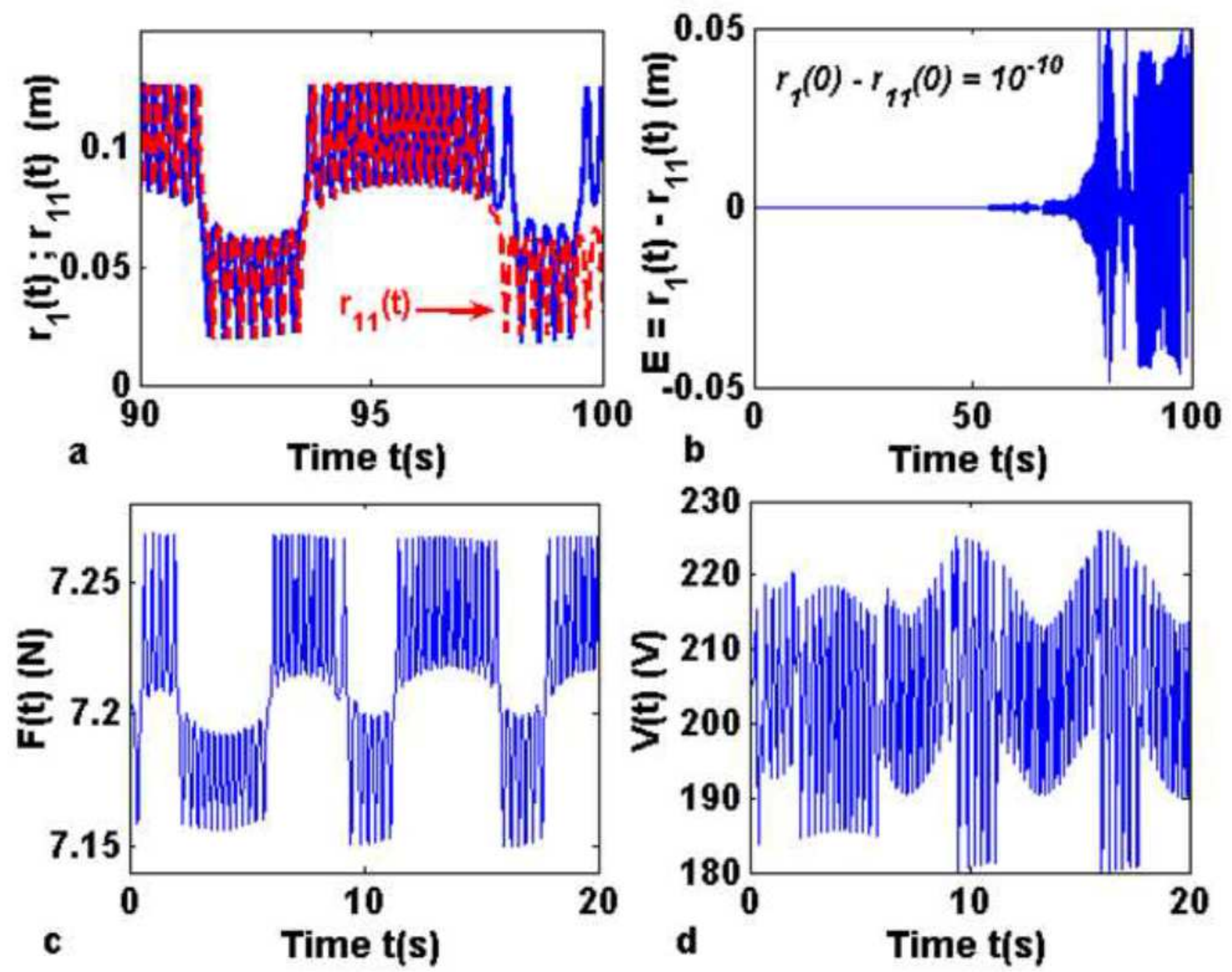

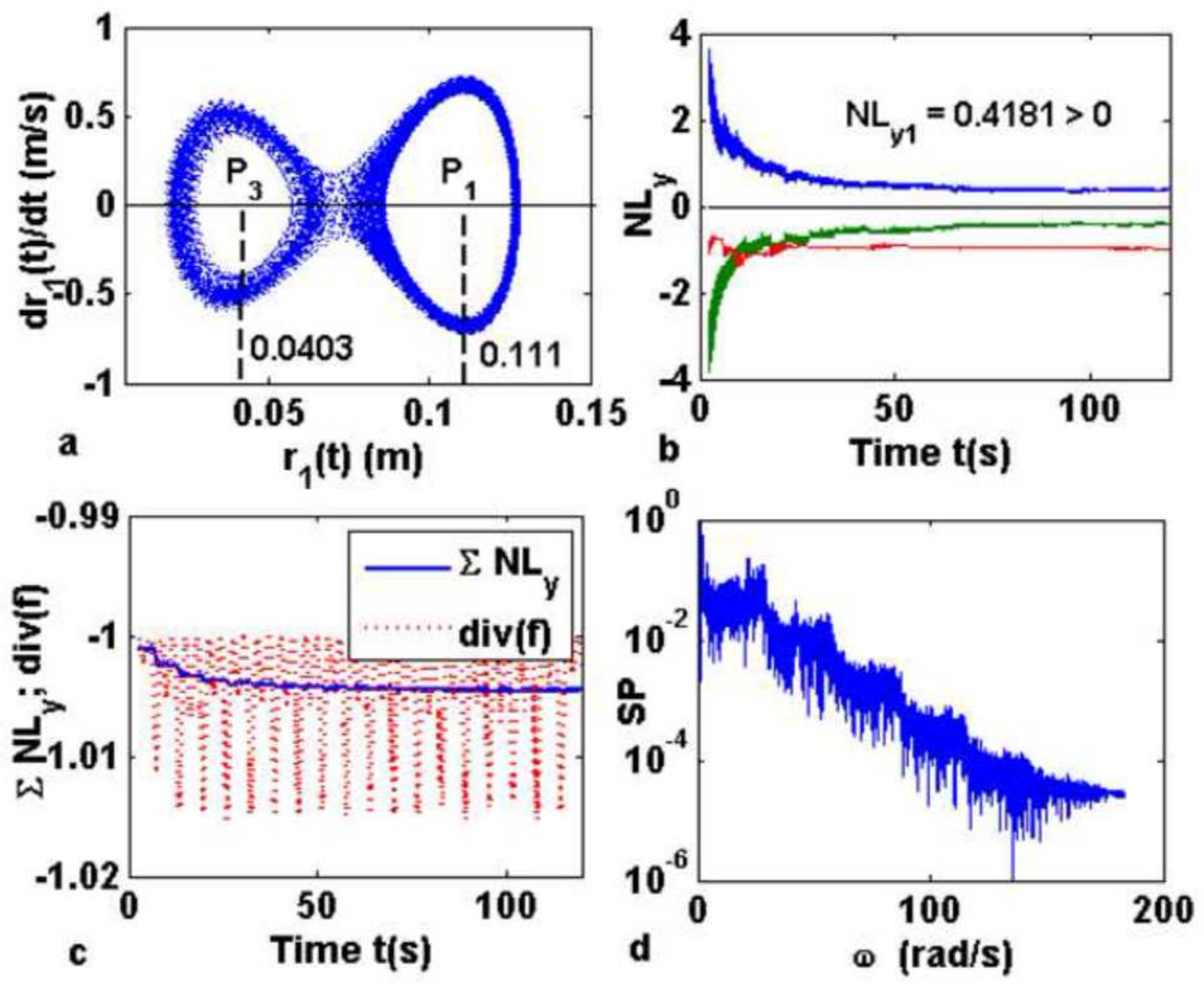


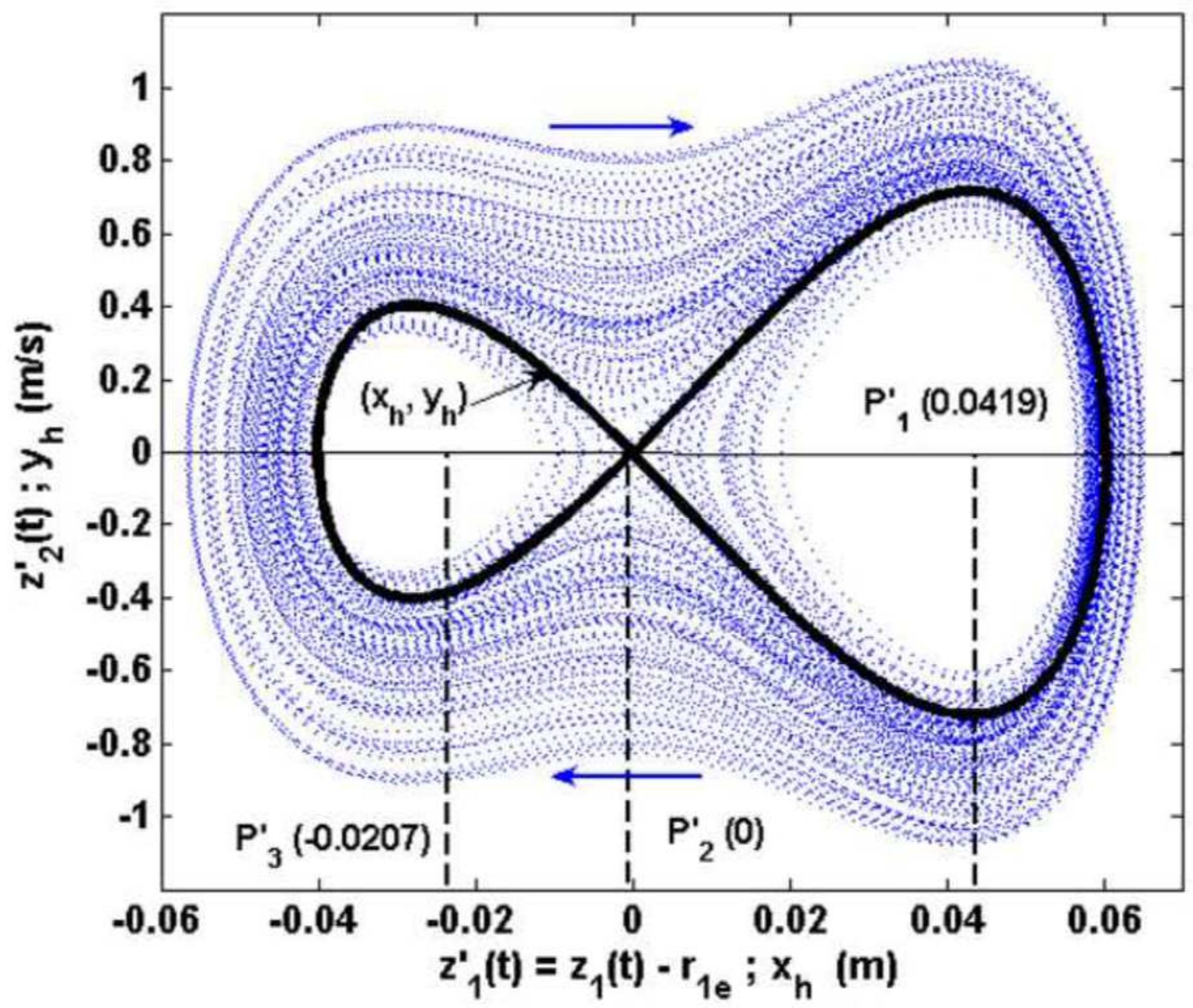



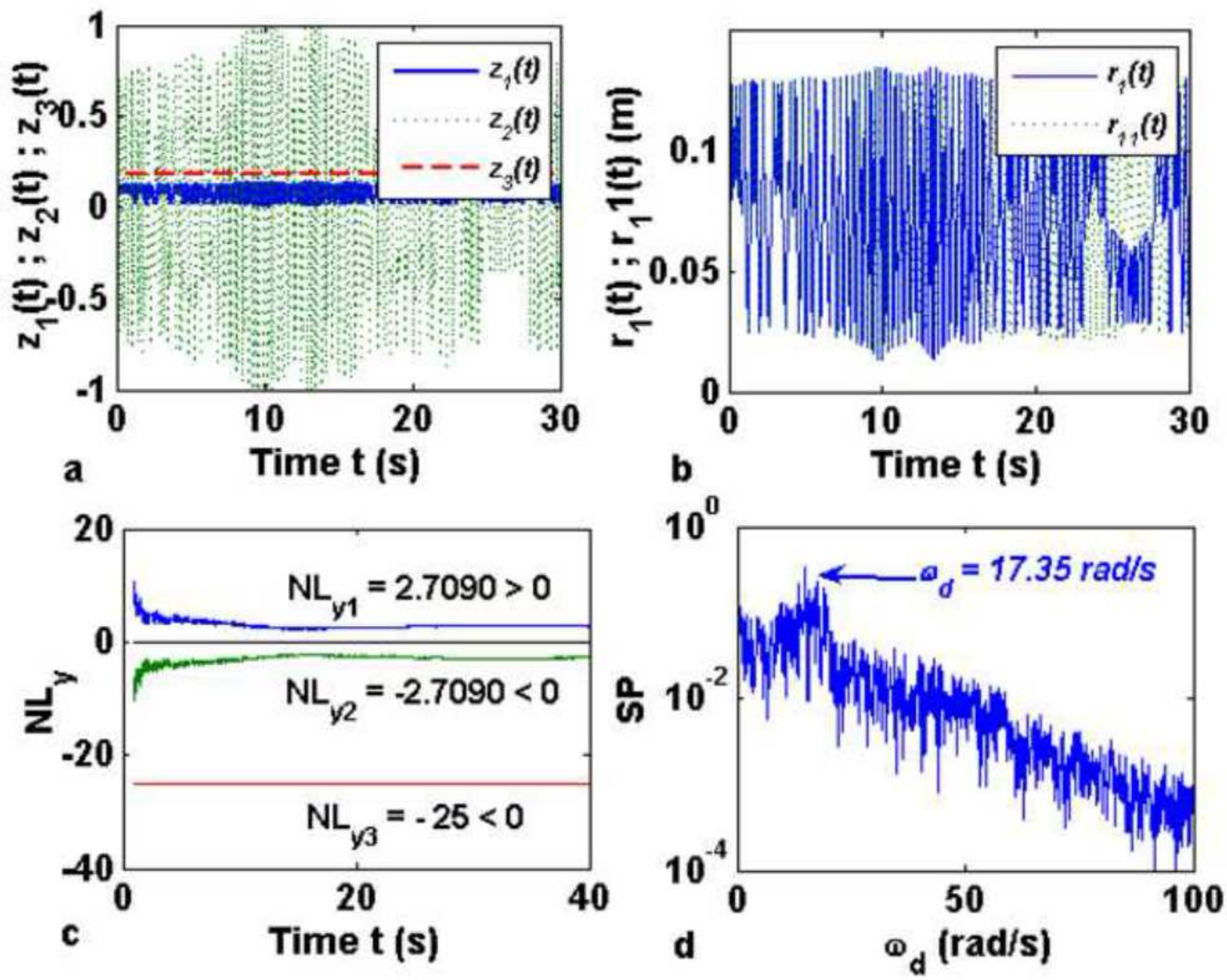

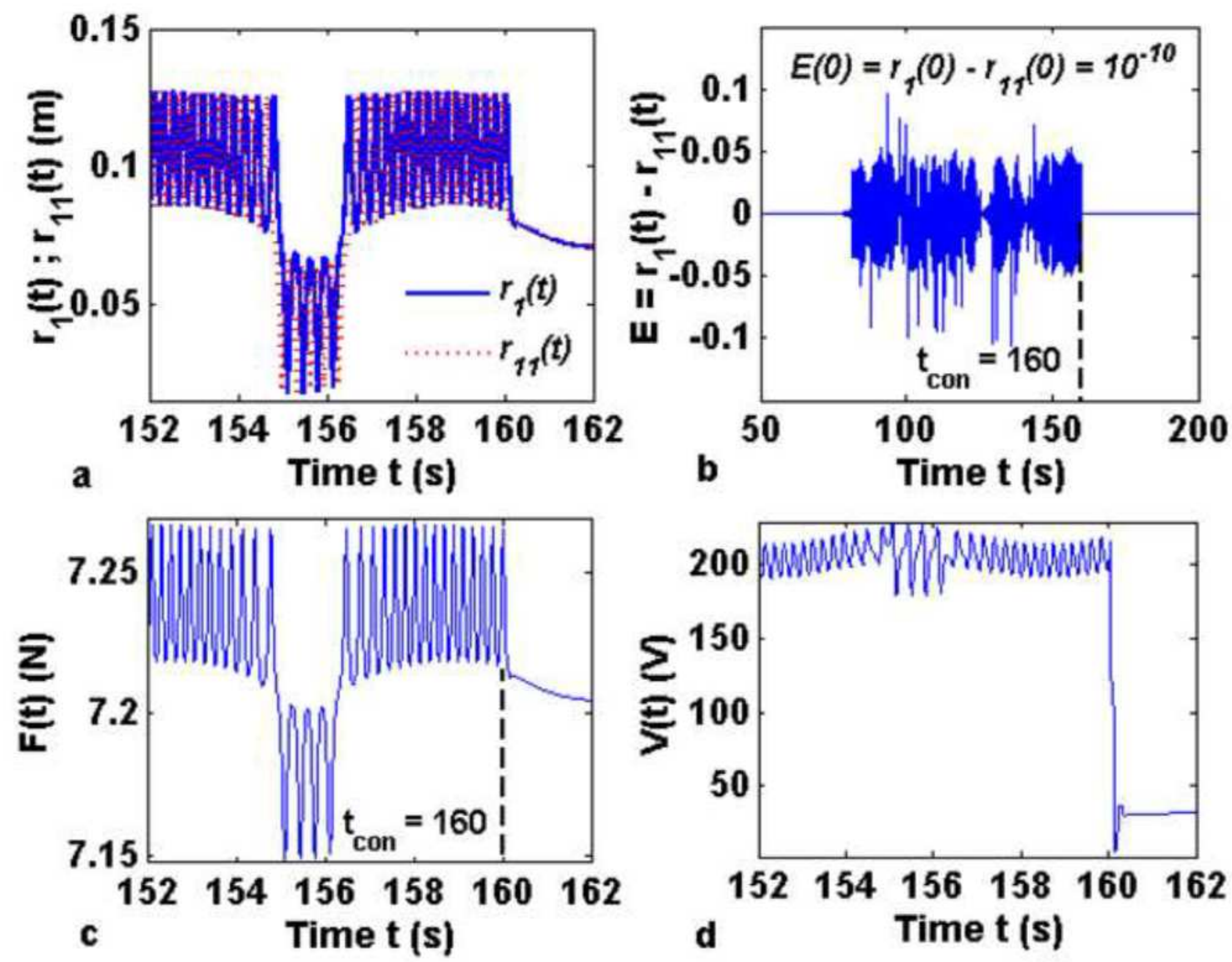

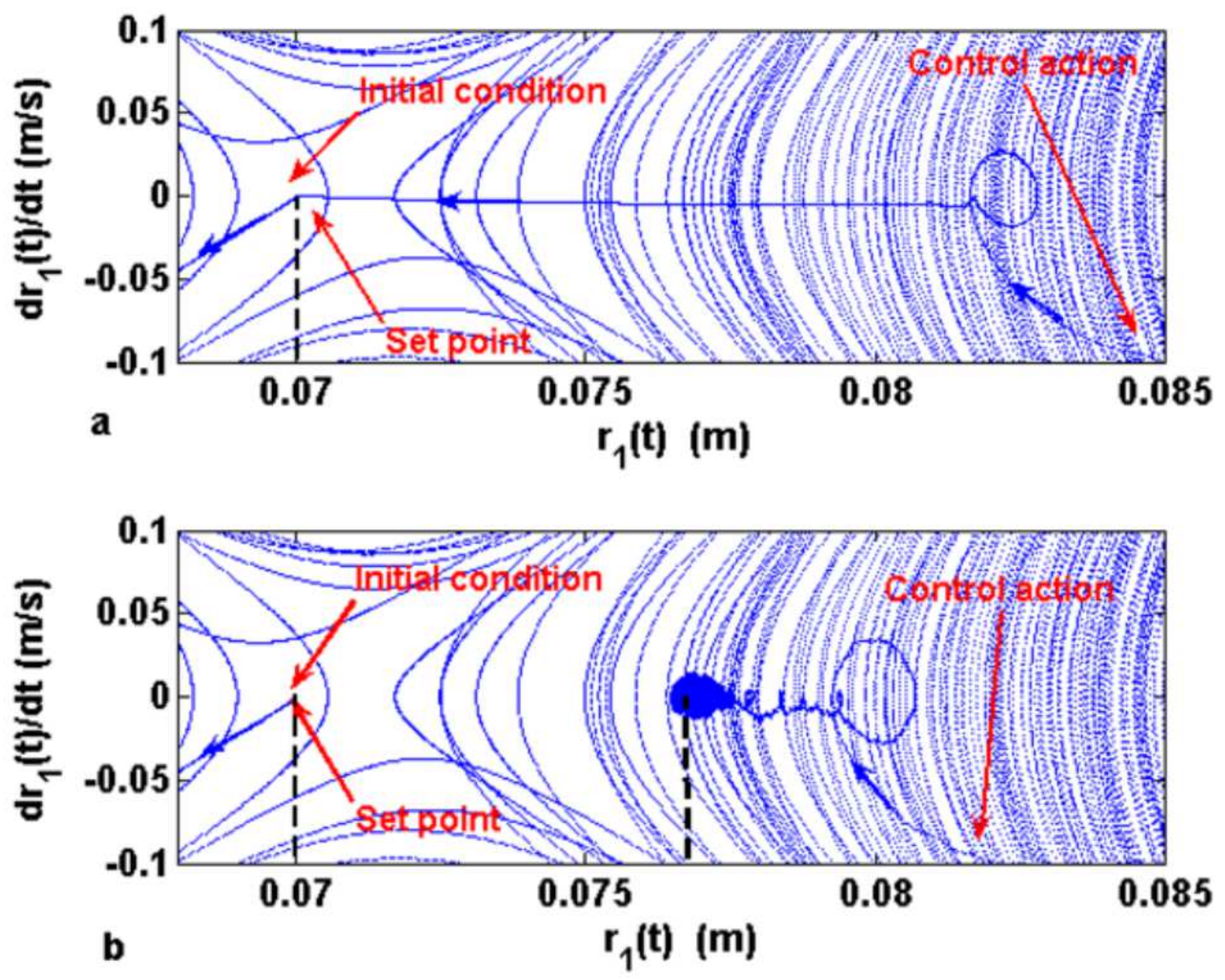

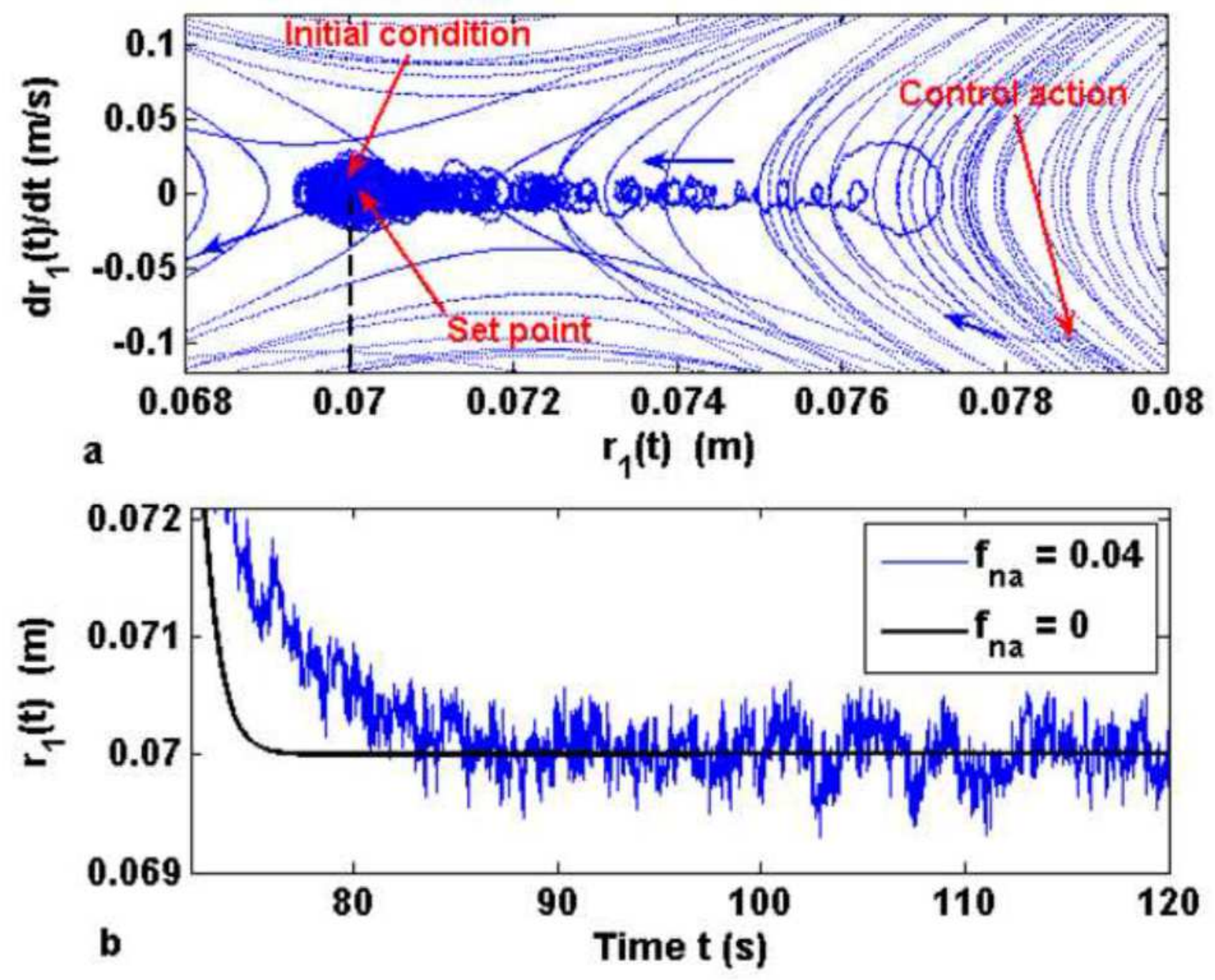


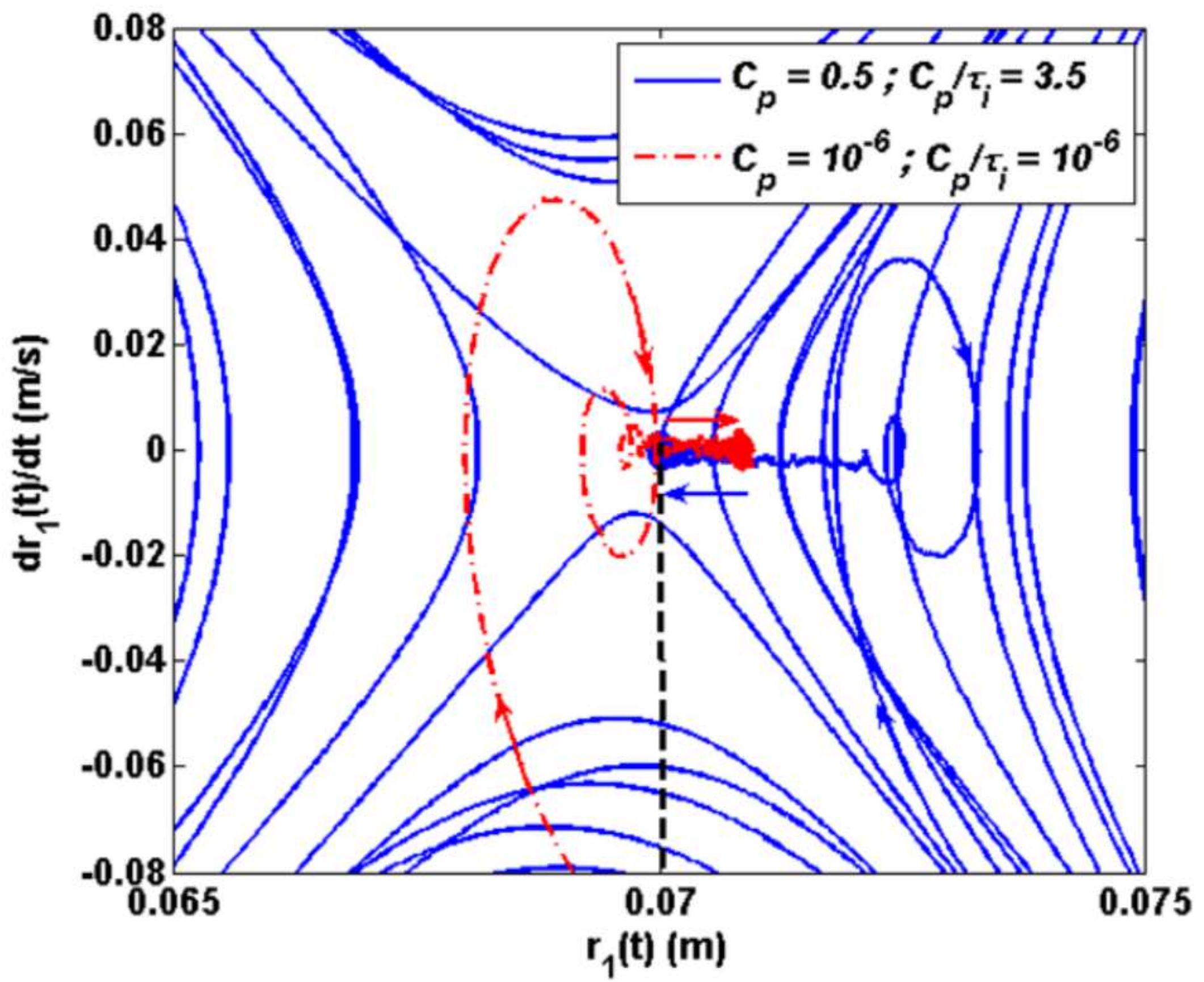

